\title{
Second order monotone finite differences discretization of linear anisotropic differential operators
}

\author{
Frédéric Bonnans* Guillaume Bonnet ${ }^{\dagger} \quad$ Jean-Marie Mirebeau ${ }^{\ddagger}$
}

March 8, 2021

\begin{abstract}
We design adaptive finite differences discretizations, which are degenerate elliptic and second order consistent, of linear and quasi-linear partial differential operators featuring both a first order term and an anisotropic second order term. Our approach requires the domain to be discretized on a Cartesian grid, and takes advantage of techniques from the field of low-dimensional lattice geometry. We prove that the stencil of our numerical scheme is optimally compact, in dimension two, and that our approach is quasi-optimal in terms of the compatibility condition required of the first and second order operators, in dimension two and three. Numerical experiments illustrate the efficiency of our method in several contexts.
\end{abstract}

\section{Introduction}

In this paper, we design finite difference discretizations of Degenerate Elliptic (DE) Partial Differential Equations (PDEs). This class of equations is sufficiently general to encompass a wide variety of applications, in the fields of optimal transport, game theory, differential geometry, stochastic modeling and finance, optimal control, etc. Our results are limited to linear and quasi-linear operators, but could in principle be used as a building block for the discretization of fully non-linear operators, see Appendix A. The assumption of degenerate ellipticity yields comparison principles and stability properties [CIL92].

Discrete Degenerate Ellipticity (DDE), for numerical schemes, implies similarly strong properties [Obe06], which often turn proofs of convergence into simple verifications. A known limitation of DDE discretization schemes is their consistency order with the original PDE, which cannot exceed two for second order operators and one for first order operators [Obe06]. However, many common implementations of second order DE operators only achieve first order consistency, or sometimes less. They may also rely on excessively wide stencils, especially in the context of two-scales discretizations [FO11, LN18, LN18]. This degrades the accuracy of the numerical results, which severely constrains the practical uses of these methods. The objective of this paper is to characterize when a second order monotone discretization is feasible, and

*Inria-Saclay and CMAP, École Polytechnique, Palaiseau, France

Frédéric Bonnans acknowledges support from the Chair Finance \& Sustainable Development and of the FiME Lab (Institut Europlace de Finance).

${ }^{\dagger}$ LMO, Université Paris-Saclay, Orsay, France, and Inria-Saclay and CMAP, École Polytechnique, Palaiseau, France

${ }^{\ddagger}$ University Paris-Saclay, ENS Paris-Saclay, CNRS, Centre Borelli, F-91190 Gif-sur-Yvette, France This work was partly supported by ANR research grant MAGA, ANR-16-CE40-0014. 
how wide the numerical scheme stencil must be, especially when the second order part of the operator is strongly anisotropic, and the first order term is non-vanishing.

The finite difference schemes developed in this paper are adaptive in the sense that the stencil of the numerical scheme depends on the PDE coefficients, and reflects the strength and orientation of the anisotropy of the PDE, see Figure 1. We do however use a fixed and non-adaptive Cartesian discretization grid $\Omega \cap h \mathbb{Z}^{d}$. This understanding of adaptivity must be distinguished from the (more standard) approach where the sampling density of the set of discretization points is adjusted locally in the PDE domain, often depending on the singularities of the solution and using a-priori or a-posteriori estimators, see e.g. [DK09]. In other words, we do not in this work adapt the set of discretization points to the addressed PDE and its solution, but we adapt the connectivity between these points as defined by the numerical scheme stencils. This paper builds on [Mir16] which similarly discusses optimally compact stencils for finite differences discretizations of several PDEs - divergence form and non-divergence form anisotropic diffusion, anisotropic eikonal equation - preserving suitable structural properties, in two dimensions. Regarding the non-divergence form Laplacian, the present work differs from [Mir16] by focusing on the obstructions to discretization related to the presence of an additional first order term, and by addressing the three dimensional case.

We state our theoretical results in the context of linear operators with constant coefficients, defined over $\mathbb{R}^{d}$ where $d \in\{2,3\}$. Because degenerate ellipticity is a local property, which is stable under a variety of transformations, they admit straightforward extensions to quasilinear operators and to some fully non-linear operators. Non-constant coefficients and bounded domains with Dirichlet boundary conditions are also easily handled. See Appendix A and the numerical experiments $\S 4$ for these extensions.

We define the linear operator $\mathcal{L}=\mathcal{L}[\omega, D]$ on $\mathbb{R}^{d}$ by the expression

$$
-\mathcal{L} u(x):=\langle\omega, \nabla u(x)\rangle+\frac{1}{2} \operatorname{Tr}\left(D \nabla^{2} u(x)\right),
$$

where $\omega \in \mathbb{R}^{d}, D \in S_{d}^{++}$is a symmetric positive definite matrix, and the unknown $u: \mathbb{R}^{d} \rightarrow \mathbb{R}$ is a smooth function. Likewise in the discrete setting we define the finite differences operator $L_{h}=L_{h}\left[\rho_{i}, e_{i}\right]_{1 \leq|i| \leq I}$, on the Cartesian grid $h \mathbb{Z}^{d}$ with grid scale $h>0$, by the expression

$$
-L_{h} u(x):=h^{-2} \sum_{1 \leq|i| \leq I} \rho_{i}\left(u\left(x+h e_{i}\right)-u(x)\right),
$$

where $\rho_{-I}, \cdots, \rho_{-1}, \rho_{1}, \cdots, \rho_{I} \geq 0$ are non-negative weights, and $e_{1}, \cdots, e_{i} \in \mathbb{Z}^{d}$ are offsets with integer entries, for some positive integer $I$. Here and throughout this paper, without loss of generality, we use the convention that $e_{-i}:=-e_{i}$ for all $1 \leq i \leq I$. Note that consistency between (1.1) and (1.2) across grid scales $h>0$ requires that the weights $\left(\rho_{i}\right)_{1 \leq|i| \leq I}$ depend on $h$, in addition to $\omega$ and $D$.

Any translation invariant linear operator on $h \mathbb{Z}^{d}$, finitely supported and vanishing on constant functions, can be written in the form (1.2). We denote by $S_{d}$ the set of symmetric $d \times d$ matrices, by $S_{d}^{+}$the subset of semi-definite ones, and by $S_{d}^{++}$the positive definite ones.

Definition 1.1. The operator $\mathcal{L}[\omega, D]$ is said Degenerate Elliptic (DE) if $D \in S_{d}^{+}$. The discrete operator $L_{h}\left[\rho_{i}, e_{i}\right]_{1 \leq|i| \leq I}$ is said Discrete Degenerate Elliptic (DDE) if $\rho_{i} \geq 0$ for all $1 \leq|i| \leq I$.

In particular, the DE property does not impose any restrictions on the first order term $\omega \in \mathbb{R}^{d}$, and the DDE property does not constrain the numerical scheme offsets $e_{i} \in \mathbb{Z}^{d}$. The objective of this paper is to construct second order accurate DDE discretizations of DE operators, and to investigate the possible obstructions to do so. For that purpose we introduce the following compatibility condition. 
Definition 1.2 (Absolute feasibility). Let $\omega \in \mathbb{R}^{d}$ and $D \in S_{d}^{+}$. We say that the pair $(\omega, D)$ is absolutely feasible if there exists an integer $I \geq 0$, some integral offsets $e_{1}, \cdots, e_{I} \in \mathbb{Z}^{d} \backslash\{0\}$, and some non-negative weights $\rho_{-I}, \cdots, \rho_{-1}, \rho_{1} \cdots, \rho_{I} \geq 0$, such that denoting $e_{-i}:=-e_{i}$ for all $1 \leq i \leq I$ one has

$$
\sum_{1 \leq|i| \leq I} \rho_{i} e_{i}=\omega, \quad \sum_{1 \leq|i| \leq I} \rho_{i} e_{i} e_{i}^{\mathrm{T}}=D .
$$

Let us emphasize that in Definition 1.2 the integer $I$ and the offsets $\left(e_{i}\right)_{1 \leq|i| \leq I}$ are not fixed a-priori (and neither are the weights $\left(\rho_{i}\right)_{1 \leq|i| \leq I}$ ), but they may depend on $\omega$ and $D$; when they exist, they are in general not unique. In this paper, we fully characterize when a pair $(\omega, D)$ is absolutely feasible in dimension $d \in\{2,3\}$, see Proposition 3.9, which is not straightforward unless $D$ is a diagonal matrix. We also advocate for a specific construction, see Definition 1.5, for which the stencil cardinality $I$ is bounded in terms of the dimension $d$, whereas the stencil width $\max \left\{\left\|e_{i}\right\| ; 1 \leq i \leq I\right\}$ is bounded in terms of condition number of $D$, see Theorem 1.9 below.

Proposition 1.3. The pair $(h \omega, D)$ is absolutely feasible iff there exists $\left(\rho_{i}, e_{i}\right)_{1 \leq|i| \leq I}$ such that $\mathcal{L}[\omega, D]$ and $L_{h}\left[\rho_{i}, e_{i}\right]_{1 \leq|i| \leq I}$ are both degenerate elliptic, and are equal on all quadratic functions.

Proof. The scheme $L_{h}\left[\rho_{i}, e_{i}\right]_{1 \leq|i| \leq I}$ is well defined iff the vectors $e_{i}$ have integer coordinates, and is DDE iff the weights $\rho_{i}$ are non-negative, the same conditions that arise in Definition 1.2. For any quadratic function $u: \mathbb{R}^{d} \rightarrow \mathbb{R}$, any $e \in \mathbb{Z}^{d}$ and any $h>0$ one has the exact Taylor expansion

$$
u(x+h e)=u(x)+h\langle p, e\rangle+\frac{1}{2} h^{2}\langle e, M e\rangle,
$$

with $p:=\nabla u(x)$ and $M:=\nabla^{2} u(x)$. Therefore, using that $\langle e, M e\rangle=\operatorname{Tr}\left(M e e^{T}\right)$,

$L_{h} u(x)=\frac{1}{h^{2}} \sum_{1 \leq|i| \leq I} \rho_{i}\left(h\left\langle p, e_{i}\right\rangle+\frac{1}{2} h^{2} \operatorname{Tr}\left(M e_{i} e_{i}^{T}\right)\right)=\frac{1}{h}\left\langle p, \sum_{1 \leq|i| \leq I} \rho_{i} e_{i}\right\rangle+\frac{1}{2} \operatorname{Tr}\left(M \sum_{1 \leq|i| \leq I} \rho_{i} e_{i} e_{i}^{T}\right)$.

On the other hand $\mathcal{L} u(x)=\langle p, \omega\rangle+\frac{1}{2} \operatorname{Tr}(M D)$. The announced result follows by identification since $p \in \mathbb{R}^{d}$ and $M \in S_{d}$ are arbitrary.

Proposition 1.3 is stated in terms of the pair $(h \omega, D)$, because the multiplicative factor $h$ arises naturally in the application to finite difference schemes; in contrast, we avoid this factor in Definition 1.2 for clarity. In dimension $d=1$, and viewing 1 -vectors and $1 \times 1$-matrices as scalars, one easily checks that $(\omega, D)$ is absolutely feasible iff $|\omega| \leq D$, and with the notations of Definition 1.2 one has $I=1, e_{1}=1$, and $\rho_{ \pm 1}=\frac{1}{2}(D \pm \omega)$, which corresponds to the usual centered finite differences scheme. Note that discretizing (1.1) using upwind finite differences for the first order term fails the consistency test on quadratic functions requested in Proposition 1.3. The following construction generalizes the centered finite differences scheme to dimension $d \in\{2,3\}$.

Our numerical scheme relies on a tool from a lattice geometry known as Selling's decomposition, described in more detail in $\S 2.1$, see also [Sel74, CS92]. It associates to each positive definite matrix $D \in S_{d}^{++}$, where $d \in\{2,3\}$, a specific decomposition of the following form

$$
D=\sum_{1 \leq i \leq I} \sigma_{i} e_{i} e_{i}^{\mathrm{T}}, \quad \quad \text { where } \sigma_{i} \geq 0, e_{i} \in \mathbb{Z}^{d}, \forall 1 \leq i \leq I,
$$

and $I:=d(d+1) / 2$. Selling's decomposition has already been used in the design of difference schemes in dimension $d \in\{2,3\}$, for (divergence form) anisotropic diffusion in [FM14], and for 
various anisotropic eikonal equations in [Mir17, Mir19]. It is at the foundation of degenerate elliptic and second order consistent discretizations of the fully non-linear two dimensional MongeAmpere [BCM16] and Pucci [BBM21] equations. In dimension $d=2$, an equivalent construction based on the Stern-Brocot dyadic tree of rational numbers is used in [BOZ04] for the HamiltonJacobi-Bellman equation of Stochastic control.

The support $\left(e_{i}\right)_{i=1}^{I}$ of Selling's decomposition, which is also the stencil of the numerical scheme proposed in this paper, tends to align with the anisotropy defined by the matrix $\mathrm{D}$. This is illustrated on Figure 1, where we use the following parametrization (closely related with Pauli matrices in quantum mechanics) of the set of symmetric positive definite matrices of size two and with unit determinant:

$$
D(a, b):=\frac{1}{\sqrt{1-a^{2}-b^{2}}}\left(\begin{array}{cc}
1+a & b \\
b & 1-a
\end{array}\right), \quad a^{2}+b^{2}<1 .
$$

Definition 1.4 (Finite difference operators). For any $e \in \mathbb{Z}^{d}, h>0, u: h \mathbb{Z}^{d} \rightarrow \mathbb{R}$, we let

$$
\delta_{h}^{e} u(x):=\frac{u(x+h e)-u(x-h e)}{2 h}, \quad \Delta_{h}^{e} u(x):=\frac{u(x+h e)-2 u(x)+u(x-h e)}{h^{2}} .
$$

Given $D \in S_{d}^{++}$where $d \in\{2,3\}$, with Selling decomposition $D=\sum_{1 \leq i \leq I} \sigma_{i} e_{i} e_{i}^{\mathrm{T}}$, we let

$$
\nabla_{h}^{D} u(x)=\sum_{1 \leq i \leq I} \sigma_{i} \delta_{h}^{e_{i}} u(x) e_{i}, \quad \Delta_{h}^{D} u(x)=\sum_{1 \leq i \leq I} \sigma_{i} \Delta_{h}^{e_{i}} u(x) .
$$

The centered finite differences $\delta_{h}^{e} u(x)=\langle e, \nabla u(x)\rangle+\mathcal{O}\left(h^{2}\right)$ and second order finite differences $\Delta_{h}^{e} u(x)=\left\langle e, \nabla^{2} u(x) e\right\rangle+\mathcal{O}\left(h^{2}\right)$, are classical constructs. In combination with Selling's decomposition, they are here used to define discrete anisotropic gradient and laplacian operators, with the following consistency properties easily derived from (1.4)

$$
\nabla_{h}^{D} u(x)=D \nabla u(x)+\mathcal{O}\left(h^{2}\right), \quad \Delta_{h}^{D} u(x)=\operatorname{Tr}\left(D \nabla^{2} u(x)\right)+\mathcal{O}\left(h^{2}\right) .
$$

For context, Selling's decomposition of the matrix $D=$ Id yields up to permutation the canonical basis $\left(e_{1}, \cdots, e_{d}\right)$ with unit weights $\sigma_{1}=1, \cdots, \sigma_{d}=1$, whereas the remaining weights vanish: $\sigma_{i}=0$ for all $d<i \leq I:=d(d+1) / 2$ (and the corresponding vectors $e_{i}$ are not uniquely determined). As a result $\nabla_{h}^{\mathrm{Id}}$ and $\Delta_{h}^{\mathrm{Id}}$ are the classical finite differences discretizations of the gradient and Laplacian, whose stencil only involves the immediate grid neighbors.

Definition 1.5 (Canonical discretization). We say that $(h \omega, D) \in \mathbb{R}^{d} \times S_{d}^{++}, d \in\{2,3\}$, is canonically feasible if the following operator $L_{h}$ is DDE

$$
-L_{h} u(x):=\left\langle D^{-1} \omega, \nabla_{h}^{D} u(x)\right\rangle+\frac{1}{2} \Delta_{h}^{D} u(x) .
$$

Equivalently, but more explicitly, $(\omega, D)$ is canonically feasible iff the following weights are non-negative

$$
\rho_{i}:=\frac{\sigma_{i}}{2}\left(1+\left\langle\omega, D^{-1} e_{i}\right\rangle\right),
$$

for all $1 \leq|i| \leq I$, where $D=\sum_{1 \leq i \leq I} \sigma_{i} e_{i} e_{i}^{T}$ is Selling's decomposition (1.4) and thus $I:=$ $d(d+1) / 2$. Note that these weights obey (1.3) by construction, which reflects the fact that for a quadratic function $u$ the expansions (1.6) are exact (no remainder), so that (1.8) matches (1.1).

Definition 1.5 outlines a simple, canonical and practical discretization of the anisotropic linear PDE operator (1.1), often referred to as our numerical scheme in the paper. By construction, 

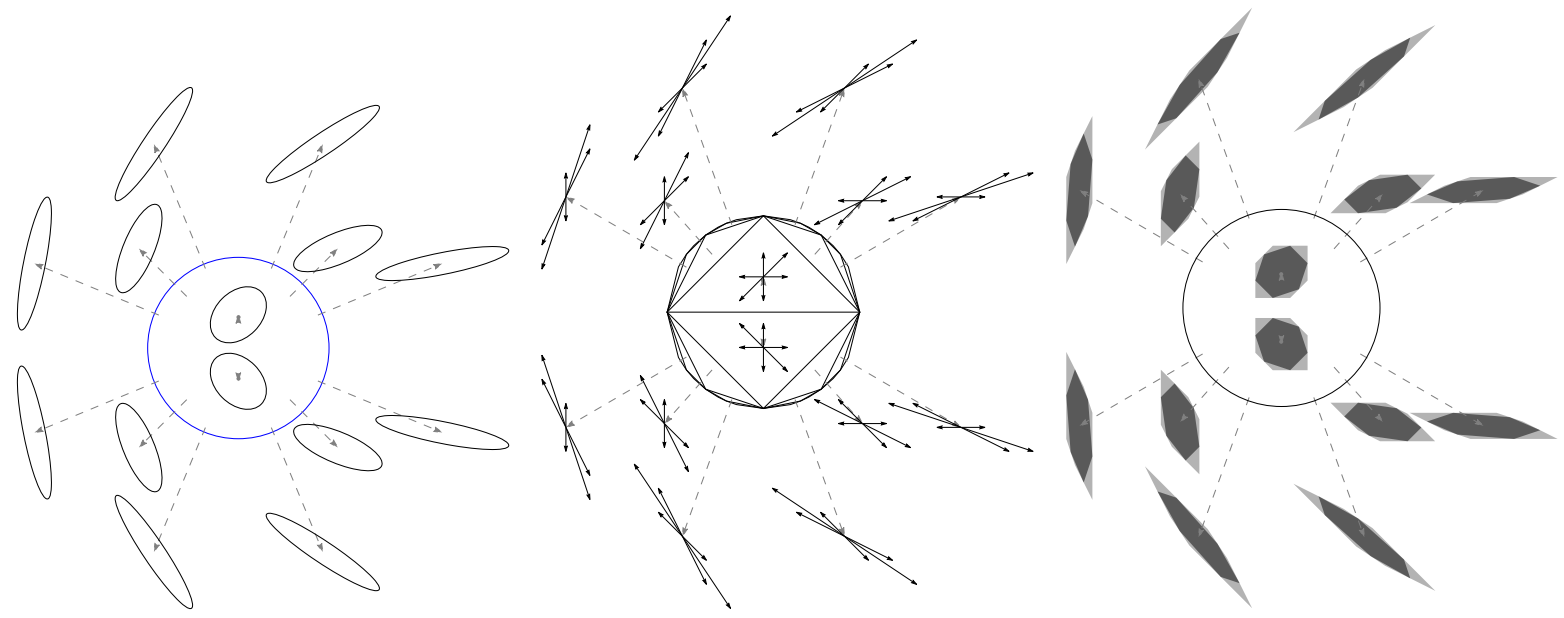

Figure 1: To each point $(a, b)$ of the unit disk, we associate the matrix $D=D(a, b)$ defined by (1.5). Left: Ellipse defined by $\left\{\left\langle v, D^{-1} v\right\rangle \leq 1 ; v \in \mathbb{R}^{2}\right\}$. Points close to the unit disk boundary (shown blue) yield strongly anisotropic ellipses. Center: Support $\left(e_{i}\right)_{i=1}^{I}$ of Selling's decomposition, which is also the stencil of our finite difference scheme for the given anisotropy. Right: Set of vectors $\omega$ for which the pair $(\omega, D)$ is canonically feasible (dark gray), or absolutely feasible (dark and light gray), computed via Proposition 3.9. The scale of the three figures may not match.

canonical feasibility implies absolute feasibility, but the latter can be achieved in a variety of other ways, using possibly a different number of terms $I$, a different support $\left(e_{i}\right)_{i=1}^{I}$, or different weights $\left(\rho_{i}\right)_{1 \leq|i| \leq I}$. Note also that (1.7) is second order consistent with the PDE operator (1.1), in view of (1.6), whereas the conditions of Definition 1.2 only imply first order consistency. We next state the main result of this paper.

Theorem 1.6. Let $(\omega, D) \in \mathbb{R}^{d} \times S_{d}^{++}$, where $d \in\{2,3\}$. If $(\omega, D)$ is absolutely feasible, then $\left(c_{d} \omega, D\right)$ is canonically feasible, with $c_{2}:=1 / 2$ and $c_{3}:=1 / 6$.

Taking the contraposition, Theorem 1.6 shows that if the canonical discretization of Definition 1.5 does not yield a DDE scheme in some practical instance, then (up to the factor $c_{d}$ ) obtaining a DDE scheme will require a serious compromise: either substantially reduce the grid scale (which amounts to multiplying $\omega$ by a small factor by homogeneity), or give up second order consistency (which allows using upwind finite differences, and eliminates the constraint of absolute feasibility). The following result in contrast provides a direct criterion for canonical feasibility. We denote by $\|\cdot\|$ and $\langle\cdot, \cdot\rangle$ the Euclidean norm and scalar product. Let also $\|e\|_{M}:=\sqrt{\langle e, M e\rangle}$ and $\|A\|:=\max _{\|x\| \leq 1}\|A x\|$ for any $e \in \mathbb{R}^{d}, M \in S_{d}^{++}$, and matrix $A$.

Theorem 1.7. Let $(\omega, D) \in \mathbb{R}^{d} \times S_{d}^{++}$, where $d \in\{2,3\}$, and let $M:=D^{-1}$. If one has

$$
\|M\|^{\frac{1}{2}}\|\omega\|_{M} \leq c_{d}
$$

then $(\omega, D)$ is canonically feasible, with $c_{2}:=1 / 2$ and $c_{3}:=1 /(4 \sqrt{3})$.

The existence of a finite differences discretization, degenerate elliptic and second order consistent, is not the only practical concern: the width of the stencil used is also of importance. Excessively wide stencils reduce the effective discretization scale of the scheme, thus also the 
accuracy of the numerical results. They may also raise difficulties with the treatment of boundary conditions, computer parallelization, matrix conditioning and sparsity, etc. See also the discussion $\S 4$. We provide two results related to the stencil width. First, we show that the canonical discretization has the smallest support of all possible DDE and second order consistent discretizations, in dimension two, in the strong sense of convex hull inclusion. We denote by $\operatorname{Hull}(E)$ the convex hull of a subset $E$ of a vector space.

Theorem 1.8. Let $(\omega, D) \in \mathbb{R}^{2} \times S_{2}^{++}$be canonically feasible, and let $\left(\rho_{i}, e_{i}\right)_{1 \leq|i| \leq I}$ be the corresponding decomposition (1.8), pruned so that $\rho_{i} \neq 0$ or $\rho_{-i} \neq 0$ for all $1 \leq i \leq I$. Let $\left(\rho_{i}^{\prime}, e_{i}^{\prime}\right)_{1 \leq|i| \leq I^{\prime}}$ be another decomposition, as in Definition 1.2. Then

$$
\operatorname{Hull}\left\{e_{i} ; 1 \leq|i| \leq I\right\} \subseteq \operatorname{Hull}\left\{e_{i}^{\prime} ; 1 \leq|i| \leq I^{\prime}\right\} .
$$

Second, we provide explicit bounds on the stencil width in terms of the differential operator coefficients and anisotropy.

Theorem 1.9. Let $(\omega, D) \in \mathbb{R}^{d} \times S_{d}^{++}$be canonically feasible, where $d \in\{2,3\}$, and let $\left(\rho_{i}, e_{i}\right)_{1 \leq|i| \leq I}$ be the corresponding decomposition (1.8), where $I=d(d+1) / 2$. Then $\left\|e_{i}\right\|_{M} \leq$ $C_{d} \sqrt{\|M\|}$ for all $1 \leq i \leq I$, where $M:=D^{-1}$, and $C_{2}=2$ and $C_{3}=4 \sqrt{3}$.

Theorem 1.9 implies in particular that $\left\|e_{i}\right\| \leq C_{d} \operatorname{Cond}(D)$, for all $1 \leq i \leq I$, where $\operatorname{Cond}(D):=\sqrt{\|D\|\left\|D^{-1}\right\|}$. See also [Mir16] for average case bounds in dimension $d=2$, under random rotations $R_{\theta}^{\mathrm{T}} D R_{\theta}$ of the tensor, $\theta \in[0,2 \pi]$.

\section{Outline}

Section $\S 2$ is devoted to further discussion of the canonical discretization, and to the proofs of Theorems 1.7, 1.8 and 1.9 which follow rather directly from arguments presented in [Mir17] and [Mir16]. Section $\S 3$ establishes Theorem 1.6. Numerical experiments are presented in $\S 4$.

\section{The canonical discretization}

This section is devoted to a further presentation of the construction of Definition 1.5, here referred to as the canonical discretization of a second order linear PDE operator. We review Selling's algorithm in $§ 2.1$, finalizing the algorithmic description of our numerical scheme. We describe in $\S 2.2$ an interpretation of this algorithm as an optimization procedure, involving objects from the field of lattice geometry known as Voronoi's first reduction and Ryskov's polyhedron. Theorems 1.7, 1.8 and 1.9 are proved in $§ 2.3$.

The results presented $\S 2.3$ are new, whereas the more classical techniques described in $\S 2.1$ and $\S 2.2$ are required for completeness and as a preliminary to the proof of Theorem 1.6 in $\S 3$.

\subsection{Selling's algorithm and formula}

We describe Selling's algorithm [Sel74, CS92], and the related tensor decomposition formula which is invoked in Definition 1.5 of the numerical scheme considered in this paper.

\section{Selling's algorithm}

This algorithm belongs to the field of lattice geometry [NS04], which among other things studies coordinate systems in additive lattices (here $\mathbb{Z}^{d}$ ), adapted to the geometry defined by a given positive definite quadratic form (here defined by $D \in S_{d}^{++}$). The next definition introduces such a concept. 
Definition 2.1. A superbase of $\mathbb{Z}^{d}$ is a $(d+1)$-tuple $b=\left(v_{0}, \cdots, v_{d}\right) \in\left(\mathbb{Z}^{d}\right)^{d+1}$ such that $\left|\operatorname{det}\left(v_{1}, \cdots, v_{d}\right)\right|=1$ and $v_{0}+\cdots+v_{d}=0$. It is said $D$-obtuse, where $D \in S_{d}^{++}$, if $\left\langle v_{i}, D v_{j}\right\rangle \leq 0$ for all $0 \leq i<j \leq d$.

Given a positive definite tensor $D \in S_{d}^{++}$, where $d \in\{2,3\}$, Selling's algorithm constructs a $D$-obtuse superbase, see Algorithm 1. Note that the algorithm does not extend to dimension $d \geq 4$, and indeed there exists a matrix $D \in S_{4}^{++}$for which no $D$-obtuse superbase exists [Sch09a].

Algorithm 1 Selling's algorithm

Input: A positive definite tensor $D \in S_{d}^{++}$, and a superbase $b=\left(v_{0}, \cdots, v_{d}\right)$, where $d \in\{2,3\}$. While there exists $0 \leq i<j \leq d$ such that $\left\langle v_{i}, D v_{j}\right\rangle>0$ do

$$
\begin{aligned}
& \text { If } d=2, b \leftarrow\left(-v_{i}, v_{j}, v_{i}-v_{j}\right) . \\
& \text { If } d=3, b \leftarrow\left(-v_{i}, v_{j}, v_{i}+v_{k}, v_{i}+v_{l}\right) \text { where }\{k, l\}=\{0,1,2,3\} \backslash\{i, j\} .
\end{aligned}
$$

Output: $b$, which is now a $D$-obtuse superbase.

Proof of correctness and termination of Algorithm 1. Denote by $b$ the current superbase at the beginning of an iteration. If the stopping criterion holds, then $b$ is $D$-obtuse, as desired. Otherwise, denoting by $b^{\prime}$ the updated superbase, one easily checks that

$$
\mathcal{E}_{D}\left(b^{\prime}\right)=\mathcal{E}_{D}(b)-C_{d}\left\langle v_{i}, D v_{j}\right\rangle \quad \text { where } \quad \mathcal{E}_{D}(b):=\sum_{0 \leq k \leq d}\left\|v_{k}\right\|_{D}^{2},
$$

and where $C_{2}=4$ and $C_{3}=2$. Thus $\mathcal{E}_{D}\left(b^{\prime}\right)<\mathcal{E}_{D}(b)$. Since there exists only finitely many superbases $b$ such that $\mathcal{E}_{D}(b)$ is below a given constant, Selling's algorithm must terminate.

Selling's algorithm is not the only means to produce a $D$-obtuse superbase. For instance Corollary 1 and Proposition 1 in [FM14] show in dimension $d \in\{2,3\}$ how to produce a $D$ obtuse superbase from another type of system of coordinates referred to as $D$-reduced basis, resulting in a $\mathcal{O}\left(\ln \left(\|D\|\left\|D^{-1}\right\|\right)\right)$ numerical complexity [NS04]. Selling's algorithm is however efficient enough for applications to PDE discretization, which usually involve moderate condition numbers, and therefore it is used in all our numerical experiments $\S 4$.

\section{Selling's decomposition}

This mathematical formula allows, once a $D$-obtuse superbase of $\mathbb{Z}^{d}$ is known, to decompose the tensor $D \in S_{d}^{++}$in the form of (1.4). For that purpose, we associate to each superbase a family of vectors $\left(e_{i j}\right)_{i \neq j}$ defined by duality relations.

Definition 2.2. Let $b=\left(v_{0}, \cdots, v_{d}\right)$ be a superbase of $\mathbb{Z}^{d}$. Then for any $i, j$ in $\{0, \cdots, d\}$ such that $i \neq j$ we let $e_{i j} \in \mathbb{Z}^{d}$ be the unique vector obeying $\left\langle e_{i j}, v_{k}\right\rangle:=\delta_{i k}-\delta_{j k}$, for all $0 \leq k \leq d$.

Note that Definition 2.2 characterizes $e_{i j} \in \mathbb{R}^{d}$ by $d+1$ linear relations. This does make sense in view of the redundancy $v_{0}+\cdots+v_{d}=0$ of the linear forms, and of the compatibility $\left(\delta_{i 0}-\delta_{j 0}\right)+\cdots+\left(\delta_{i d}-\delta_{j d}\right)=1-1=0$ of the right-hand sides. The vectors $e_{i j}$ admit explicit expressions when $d \in\{2,3\}$, namely (up to the sign)

$$
e_{i j}= \pm v_{k}^{\perp} \text { if } d=2, \quad\left(\text { resp. } e_{i j}= \pm v_{k} \times v_{l} \text { if } d=3\right),
$$


where $\{i, j, k\}=\{0,1,2\}$ (resp. $\{i, j, k, l\}=\{0,1,2,3\}$ ). For all $i, j, k, l \in\{0, \cdots, d\}$ such that $i \neq j$ and $k \neq l$ one also has the useful identity

$$
\begin{aligned}
\left\langle v_{k},\left(e_{i j} e_{i j}^{\mathrm{T}}\right) v_{l}\right\rangle & =\left\langle e_{i j},\left(v_{k} \otimes v_{l}\right) e_{i j}\right\rangle=\left\langle e_{i j}, v_{k}\right\rangle\left\langle e_{i j}, v_{l}\right\rangle \\
& =\left\{\begin{array}{l}
-1 \text { if }\{i, j\}=\{k, l\}, \\
0 \text { otherwise. }
\end{array}\right.
\end{aligned}
$$

We denoted by $v \otimes w:=\frac{1}{2}\left(v w^{\mathrm{T}}+w v^{\mathrm{T}}\right) \in S_{d}$ the symmetrized outer product of two vectors $v, w \in \mathbb{R}^{d}$. The next lemma shows how a superbase of $\mathbb{Z}^{d}$ defines a decomposition of an arbitrary tensor $D$, involving integer offsets. If the superbase is $D$-obtuse, then the weights are nonnegative, and the decomposition is known as Selling's decomposition or formula [Sel74, CS92].

Lemma 2.3 (Selling's decomposition). Let $D \in S_{d}$, and let $b=\left(v_{0}, \cdots, v_{d}\right)$ be a superbase of $\mathbb{Z}^{d}$. Then

$$
D=-\sum_{0 \leq i<j \leq d}\left\langle v_{i}, D v_{j}\right\rangle e_{i j} e_{i j}^{\mathrm{T}}
$$

If $D \in S_{d}^{++}$and $b$ is D-obtuse, then (2.4) is known as Selling's decomposition of $D$.

Proof. Denote by $D^{\prime}$ the r.h.s. of (2.4). By (2.3) we obtain $\left\langle v_{k}, D v_{l}\right\rangle=\left\langle v_{k}, D^{\prime} v_{l}\right\rangle$ for all $0 \leq k<$ $l \leq d$. These $d(d+1) / 2$ independent linear relations imply $D=D^{\prime}$, as announced.

We finally complete the description of our numerical scheme construction, see Definition 1.5. Given a positive definite tensor $D$, build a $D$-obtuse superbase using Selling's algorithm or another method. Then Selling's formula (2.4) yields the required tensor decomposition $D=$ $\sum_{1 \leq i \leq I} \sigma_{i} e_{i} e_{i}^{\mathrm{T}}$ with $I=d(d+1) / 2, \sigma_{i} \geq 0, e_{i} \in \mathbb{Z}^{d} \backslash\{0\}$. We emphasize that one cannot replace Selling's formula with another tensor decomposition in Definition 1.5, or Theorems 1.6, 1.7, 1.8 and 1.9 would fail. Finally, let us mention that Selling's decomposition is uniquely determined by the tensor $D$, and thus independent of the choice of $D$-obtuse superbase, see Remark 2.13.

\subsection{Ryskov's polyhedron and Voronoi's first reduction}

We introduce two concepts from lattice geometry, Ryskov's polyhedron and Voronoi's first reduction [Sch09a], allowing us to rephrase Selling's algorithm as a a simplex-like optimization method solving a linear program. In order to prevent any confusion, let us insist that these geometric tools are not connected with the classical concept of Voronoi diagram, which is instead related with Voronoi's second reduction [Sch09a]. Ryskov's polyhedron is an unbounded subset $\mathcal{M}_{d} \subseteq S_{d}$, defined as follows ${ }^{1}$

$$
\mathcal{M}_{d}:=\left\{M \in S_{d} ; \forall e \in \mathbb{Z}^{d} \backslash\{0\},\langle e, M e\rangle \geq 1\right\} .
$$

Remark 2.4 (Identification of duplicate constraints). The constraints associated in (2.5) with a vector $e \in \mathbb{Z}^{d} \backslash\{0\}$ and with its opposite $-e$ are obviously equivalent. We regard them as a single constraint, associated with the equivalence class $\pm e$.

The main result proved in this subsection is the classification of the edges and vertices of the polyhedron $\mathcal{M}_{d}$ in dimension $d \in\{2,3\}$, see Corollary 2.11. These objects are actually known in all dimensions $d \leq 8$ see [Sch09b, CS88, SSV07], hence the results presented in this subsection are not new. The proof is recalled for completeness and because its arguments are adapted in $\S 3.1$ for the proof of Theorem 1.6.

\footnotetext{
${ }^{1}$ Depending on the author, Ryskov's polyhedron (2.5) is defined via the constraints $\langle e, M e\rangle \geq \lambda, e \in \mathbb{Z}^{d} \backslash\{0\}$, where $\lambda$ is one, two, or an unspecified positive constant [Sch09b]. These definitions are equivalent up to an homothety of $\mathcal{M}_{d}$.
} 


\section{Regularity of Ryskov's polyhedron}

We refer to Appendix B for some general terminology on polyhedra and linear programming. Recall that, by Minkowski's convex body theorem [Sch09a], any centrally symmetric convex body $K \subseteq \mathbb{R}^{d}$ of volume $\operatorname{Vol}(K)>2^{d}$ contains a point of $\mathbb{Z}^{d} \backslash\{0\}$.

Lemma 2.5. Each $M \in \mathcal{M}_{d}$ is positive definite, and $\operatorname{det}(M) \geq c_{d}$ where $c_{d}>0$ is a constant.

Proof. If $M \in \mathcal{M}_{d}$, then by construction $M$ is positive semi-definite and the set $K=\{x \in$ $\left.\mathbb{R}^{d} ;\langle x, M x\rangle<1\right\}$ contains no point of $\mathbb{Z}^{d} \backslash\{0\}$. By Minkowski's convex body theorem one has $2^{d} \geq \operatorname{Vol}(K)=\operatorname{Vol}(B) \operatorname{det}(M)^{-\frac{1}{2}}$, where $B$ denotes the Euclidean unit ball, as announced. The announced result thus holds with (sub-optimal) constant $c_{d}:=\operatorname{Vol}(B)^{2} / 2^{2 d}$.

The optimal constant in Lemma 2.5 is $c_{d}=\gamma_{d}^{-d}$, where $\gamma_{d}$ is known as Hermite's constant [Sch09a].

Corollary 2.6. The polyhedron $\mathcal{M}_{d}$ is regular in the sense of Definition B.1.

Proof. Let us check the three points of this definition. (i) The set $\mathcal{M}_{d}$ contains all $M \in S_{d}$ such that $M \succeq \mathrm{Id}$, hence it has non-empty interior, as required. (ii) The defining constraints obey $\operatorname{Span}\left\{e e^{\overline{\mathrm{T}}} ; e \in \mathbb{Z}^{d} \backslash\{0\}\right\}=\mathbb{S}_{d}$, as required. (iii) For any $M, M^{\prime} \in S_{d}$ and any $e \in \mathbb{R}^{d}$ one has $\left\langle e, M^{\prime} e\right\rangle \geq\left(\lambda_{\min }(M)-\left\|M-M^{\prime}\right\|\right)\|e\|^{2}$, where $\lambda_{\min }(M)>0$ denotes the smallest eigenvalue. Given $M \in S_{d}^{++}$, one thus has $\left\langle e, M^{\prime} e\right\rangle>1$ whenever $\left\|M^{\prime}-M\right\|<\lambda_{\min }(M) / 2$ and $\|e\| \geq 2 / \lambda_{\min }(M)$. This shows that only finitely many constraints defining the polyhedron $\mathcal{M}_{d}$ active in the neighborhood of any $M \in \mathcal{M}_{d}$, as required.

\section{Vertices and edges of Ryskov's polyhedron}

We describe a family of vertices of $\mathcal{M}_{d}$ in Lemma 2.8, the corresponding edges in Lemma 2.10, $d \in\{2,3\}$, and show in Corollary 2.11 that this exhausts the skeleton of $\mathcal{M}_{d}$.

Definition 2.7. To each superbase $b=\left(v_{0}, \cdots, v_{d}\right)$ of $\mathbb{Z}^{d}$ one associates the matrix

$$
M_{b}=\frac{1}{2} \sum_{0 \leq i \leq d} v_{i} v_{i}^{\mathrm{T}} .
$$

Lemma 2.8. Let $b=\left(v_{0}, \cdots, v_{d}\right)$ be a superbase of $\mathbb{Z}^{d}$. Then $\left\langle e, M_{b} e\right\rangle \geq 1$ for all $e \in \mathbb{Z}^{d} \backslash\{0\}$, with equality iff $e=e_{i j}$ for some $i, j \in\{0, \cdots, d\}, i \neq j$, see Definition 2.2.

Proof. Let $e \in \mathbb{Z}^{d} \backslash\{0\}$ and $S:=2\left\langle e, M_{b} e\right\rangle=\sum_{0 \leq i \leq d}\left\langle v_{i}, e\right\rangle^{2}$. Then $S$ is the sum of the squares of the integers $\left\langle v_{i}, e\right\rangle, 0 \leq i \leq d$, which are not all zero, and obey $\sum_{0 \leq i \leq d}\left\langle v_{i}, e\right\rangle=\langle 0, e\rangle=0$. Thus $S \geq 2$, with equality iff there exists $i \neq j$ such that $\left\langle v_{i}, e\right\rangle=1,\left\langle v_{j}, e\right\rangle=-1$, and $\left\langle v_{k}, e\right\rangle=0$ for all $k \notin\{i, j\}$. In other words $e=e_{i j}$, as announced.

By Lemma 2.8, one has $M_{b} \in \mathcal{M}_{d}$ for any superbase $b$. Furthermore, $M_{b}$ saturates the $d(d+1) / 2=\operatorname{dim}\left(S_{d}\right)$ linearly independent constraints associated with the vectors $\pm e_{i j}$, where $0 \leq i<j \leq d$, and satisfies strictly the constraints associated with any other $e \in \mathbb{Z}^{d} \backslash\{0\}$. This shows that $M_{b}$ is a non-degenerate vertex of the polyhedron $\mathcal{M}_{d}$. The edges emanating from this vertex, in dimension $d \in\{2,3\}$, are described in Lemma 2.10 below.

We introduce in the next definition an adjacency relation on the set of superbases of $\mathbb{Z}^{d}$, which is reminiscent of the superbase updates involved in Selling's algorithm, Algorithm 1. This similarity is not by accident, and it leads to an interpretation of Selling's algorithm as a linear program solver, see Proposition 2.12. 
Definition 2.9. One defines the following adjacency relations for superbases of $\mathbb{Z}^{d}, d \in\{2,3\}$,

$$
\begin{aligned}
\left(v_{0}, v_{1}, v_{2}\right) & \leftrightarrow\left(-v_{0}, v_{1}, v_{0}-v_{1}\right), \\
\left(v_{0}, v_{1}, v_{2}, v_{3}\right) & \leftrightarrow\left(-v_{0}, v_{1}, v_{2}+v_{0}, v_{3}+v_{0}\right),
\end{aligned}
$$

and likewise up to a permutation and/or a global change of sign of the superbase.

Lemma 2.10. Let $d \in\{2,3\}$ and let $b$ be a superbase of $\mathbb{Z}^{d}$. The edges of $\mathcal{M}_{d}$ containing $M_{b}$ coincide with the segments $\left[M_{b}, M_{b^{\prime}}\right]$, where $b^{\prime}$ is a superbase of $\mathbb{Z}^{d}$ adjacent to $b$.

Proof. Recall that $M_{b}$ is a non-degenerate vertex of $\mathcal{M}_{d}$. Therefore there exists $d(d+1) / 2=$ $\operatorname{dim}\left(S_{d}\right)$ edges of $\mathcal{M}_{d}$ containing $M_{b}$, which are obtained by relaxing one of the constraints active at $M_{b}$ (see also $\S$ B.3 on this topic). In other words, the edges of $\mathcal{M}_{d}$ containing $M_{b}$ can be parametrized by $0 \leq \alpha<\beta \leq d$ and obtained as

$$
E_{\alpha \beta}=\left\{M \in \mathcal{M}_{d} ;\left\langle e_{i j}, M e_{i j}\right\rangle=1,0 \leq i<j \leq d,(i, j) \neq(\alpha, \beta)\right\} .
$$

Let $b, b^{\prime}$ be superbases of $\mathbb{Z}^{d}$ as in (2.7). Then distinguishing dimensions we compute

$$
\begin{aligned}
(d=2): & 2\left(M_{b^{\prime}}-M_{b}\right)=\left(v_{0}-v_{1}\right)\left(v_{0}-v_{1}\right)^{\mathrm{T}}-v_{2} v_{2}^{\mathrm{T}}=-4 v_{0} \otimes v_{1} . \\
(d=3): & 2\left(M_{b^{\prime}}-M_{b}\right) \\
& =\left(v_{2}+v_{0}\right)\left(v_{2}+v_{0}\right)^{\mathrm{T}}+\left(v_{3}+v_{0}\right)\left(v_{3}+v_{0}\right)^{\mathrm{T}}-v_{2} v_{2}^{\mathrm{T}}-v_{3} v_{3}^{\mathrm{T}} \\
& =2 v_{0} \otimes\left(v_{0}+v_{2}+v_{3}\right)=-2 v_{0} \otimes v_{1} .
\end{aligned}
$$

The symmetrized outer product $\otimes$ was introduced in (2.3). Thus elements $M$ in $\left[M_{b}, M_{b^{\prime}}\right]$ obey the constraints $\left\langle e_{i j}, M e_{i j}\right\rangle=1$ whenever $\{i, j\} \neq\{0,1\}$, by (2.3). Therefore $\left[M_{b}, M_{b^{\prime}}\right] \subseteq E_{01}$, and equality holds since $M_{b}$ and $M_{b^{\prime}}$ are vertices of $\mathcal{M}_{d}$ and $E_{01} \subseteq \partial \mathcal{M}_{d}$. Likewise, by permuting the indices, we obtain for all $0 \leq i<j \leq d$ an edge of $\mathcal{M}_{d}$ of the form $\left[M_{b}, M_{b^{\prime}}\right]$ where $b^{\prime}$ is adjacent to $b$, obeying all the constraints active at the non-degenerate vertex $M_{b}$ but the one associated with $\pm e_{i j}$ (previously $\pm e_{01}$ ).

Corollary 2.11. The vertices (resp. bounded edges) of Ryskov's polyhedron $\mathcal{M}_{d}$, where $d \in$ $\{2,3\}$, take the form $M_{b}$ where $b$ is a superbase of $\mathbb{Z}^{d}$ (resp. $\left[M_{b}, M_{b^{\prime}}\right]$ where $b^{\prime}$ is an adjacent superbase). There are no unbounded edges in $\partial \mathcal{M}_{d}$.

Proof. The result follows from Lemma 2.10, and the fact that the graph defined by the vertices and edges of a regular polyhedron is connected.

\section{Voronoi's first reduction}

Voronoi's first reduction $\operatorname{Vor}(D)$, of a positive definite quadratic form $D \in S_{d}^{++}$, is defined as a linear minimization problem over Ryskov's polyhedron

$$
\operatorname{Vor}(D):=\inf _{M \in \mathcal{M}_{d}} \operatorname{Tr}(D M)
$$

This linear program, in dimension $d(d+1) / 2$ and subject to infinitely many constraints, is well posed as shown by Voronoi himself [Vor08, Sch09a], in the sense that the collection of minimizers is non-empty and compact (generically it is a point) for any $D \in S_{d}^{++}$. The next proposition reproves this fact in dimension $d \in\{2,3\}$. 
Proposition 2.12. Let $D \in S_{d}^{++}$, where $d \in\{2,3\}$. Then Voronoi's first reduction is a well posed linear program, attaining its minimum at vertices $M_{b}$ of $\mathcal{M}_{d}$ associated with a $D$-obtuse superbase $b$.

Proof. By lemma 2.10, Selling's algorithm defines a walk on the graph defined by the vertices and edges of Ryskov's polyhedron. Observing that $\mathcal{E}_{D}(b)=2 \operatorname{Tr}\left(D M_{b}\right)$, see (2.1) and (2.6), we see that the next vertex selection reduces the linear program's objective function, whenever that is possible. Compare also (2.1, left) with (2.8) and (2.9). Since Selling's algorithm terminates, it solves the linear program (2.10), by the general results in $\S$ B.2. Furthermore, by Definition 2.1 , it terminates precisely when reaching a $D$-obtuse superbase, which concludes the proof.

Note that the proof of the previous proposition outlines a close relationship between Selling's algorithm and the simplex algorithm [BG15] applied to the linear program (2.10).

Remark 2.13 (Uniqueness of Selling's decomposition). Consider the decomposition (2.4) of a tensor $D \in S_{d}^{++}$, associated with a $D$-obtuse superbase $b$ (if any exists, which is only guaranteed in dimension $d \leq 3$ ). By Lemma 2.8, it can be rephrased as a set of KKT relations for the linear program (2.10) at $M_{b} \in \mathcal{M}_{d}$, see Definition B.5. Since $M_{b}$ is a non-degenerate vertex of $\mathcal{M}_{d}$, the coefficients of this KKT relation are uniquely determined, even if there is no uniqueness of the $D$-obtuse superbase, see Proposition B.6. In contrast, Voronoi's reduction (2.10) in dimension $d \geq 4$, or our variant $\widetilde{\operatorname{Vor}}(\omega, D)$ introduced $\S 3.2$ in dimension $d \geq 2$, involve polyhedra with degenerate vertices, at which the KKT relations are often non-uniquely determined.

\subsection{Proof of Theorems 1.7, 1.8 and 1.9}

Theorems 1.7 and 1.9, announced in the introduction, provide respectively a criterion for the existence of our discretization, and an estimate of the size of its support. They both follow from the next lemma, which bounds the norm of the vectors defined dually from an obtuse superbase.

Lemma 2.14 (Corollary 4.12 in [Mir17]). Let $D \in S_{d}^{++}$where $d \in\{2,3\}$. Let $b$ be a D-obtuse superbase, and let $e=e_{i j}$, for some $i, j \in\{0, \cdots, d\}$ such that $i \neq j$, see Definition 2.2. Then, denoting $C_{2}:=2$ and $C_{3}:=4 \sqrt{3}$, one has

$$
\|e\|_{M} \leq C_{d}\|M\|^{\frac{1}{2}}, \quad \text { where } M:=D^{-1} .
$$

We refer to [Mir17] for the proof of Lemma 2.14, and use this result here to establish Theorems 1.7 and 1.9 .

Proof of Theorem 1.9. Recall that the numerical scheme construction in Definition 1.5 relies on Selling's decomposition of a tensor $D \in S_{d}^{++}$, see Lemma 2.3. In particular the offsets $\left(e_{i}\right)_{1 \leq i \leq I}$ with $I=d(d+1) / 2$ appearing in Theorem 1.9, are (up to reindexing) the same as those appearing in Lemma 2.14 and there denoted $\left(e_{i j}\right)_{0 \leq i<j \leq d}$. The announced result follows from (2.11).

Proof of Theorem 1.7. Denote $M:=D^{-1}$ and let $e=e_{i j}$ for some $0 \leq i<j \leq d$, with the notations of Lemma 2.14. Then

$$
\left|\left\langle\omega, D^{-1} e\right\rangle\right|=|\langle\omega, M e\rangle| \leq\|\omega\|_{M}\|e\|_{M} \leq C_{d}\|M\|^{\frac{1}{2}}\|\omega\|_{M} .
$$

Condition (1.9) thus implies that $\left|\left\langle\omega, D^{-1} e\right\rangle\right| \leq 1$, and therefore that the weights (1.8) are non-negative, which as announced proves the absolute feasibility of $(\omega, D)$.

The rest of this section is devoted to the proof of Theorem 1.8. For that purpose, we need to introduce the geometrical concept of Voronoi vector [Sch09a]. 
Definition 2.15. A point $e \in \mathbb{Z}^{d} \backslash\{0\}$ is an $M$-Voronoi vector, where $M \in S_{d}^{++}$, if there exists $p \in \mathbb{R}^{d}$ (referred to as the witness) such that

$$
\|p-0\|_{M}=\|p-e\|_{M} \leq\|p-x\|_{M}, \text { for all } x \in \mathbb{Z}^{d} .
$$

One says that $e$ is a strict $M$-Voronoi vector if the above inequality is strict for all $x \notin\{0, e\}$.

The origin 0 is introduced in $(2.12$, left) to emphasize the geometrical interpretation. In the language of Voronoi diagrams, $e$ is a an $M$-Voronoi vector iff the Voronoi cells of 0 and $e$ intersect, in the diagram of $\mathbb{R}^{d}$ associated with the sites $\mathbb{Z}^{d}$ and metric $\|\cdot\|_{M}$. The (strict) $M$ Voronoi vectors can be determined from an $M$-obtuse superbase, as shown by the next lemma in dimension $d=2$. See Theorem 3 in [CS92] for a related argument in arbitrary dimension.

Lemma 2.16. Let $M \in S_{2}^{++}$and let $e_{0}, e_{1}, e_{2}$ be an $M$-obtuse superbase. Then $\pm e_{0}, \pm e_{1}, \pm e_{2}$ are $M$-Voronoi vectors. Furthermore $e_{0}$ is a strict $M$-Voronoi vector iff $\left\langle e_{1}, M e_{2}\right\rangle<0$ (likewise for $-e_{0}$, and likewise permuting $\left.\left(e_{0}, e_{1}, e_{2}\right)\right)$.

Proof. We first show, w.l.o.g., that $e_{0}$ is an $M$-Voronoi vector, whose witness is $p:=e_{0} / 2$. Note that $\|p-0\|_{M}=\left\|p-e_{0}\right\|_{M}\left(=\left\|e_{0} / 2\right\|_{M}\right)$ as required (2.12). Let $x \in \mathbb{Z}^{2}$ be arbitrary. Since $\operatorname{det}\left(e_{1}, e_{2}\right)=1$, there exists $a, b \in \mathbb{Z}$ such that $x=a e_{1}+b e_{2}$. From this point a direct computation yields $(2.12)$, as announced

$$
\begin{aligned}
\|p-x\|_{M}^{2}-\|p\|_{M}^{2} & =\left\|(a+1 / 2) e_{1}+(b+1 / 2) e_{2}\right\|_{M}^{2}-\left\|\left(e_{1}+e_{2}\right) / 2\right\|_{M}^{2} \\
& =\left(a^{2}+a\right)\left\|e_{1}\right\|_{M}^{2}+\left(b^{2}+b\right)\left\|e_{2}\right\|_{M}^{2}+(2 a b+a+b)\left\langle e_{1}, M e_{2}\right\rangle \\
& \geq\left(\left(a^{2}+a\right)+\left(b^{2}+b\right)-(2 a b+a+b)\right)\left(-\left\langle e_{1}, M e_{2}\right\rangle\right) \\
& =-(a-b)^{2}\left\langle e_{1}, M e_{2}\right\rangle \geq 0 .
\end{aligned}
$$

In the third line we used $\left\|e_{1}\right\|_{M}^{2}=\left\langle-e_{0}-e_{2}, M e_{1}\right\rangle \geq-\left\langle e_{1} M e_{2}\right\rangle$, and likewise $\left\|e_{2}\right\|_{M}^{2} \geq$ $-\left\langle e_{1} M e_{2}\right\rangle$. In the rest of the proof, we show that $e_{0}$ is a strict $M$-Voronoi vector, under the additional assumption that $\left\langle e_{1}, M e_{2}\right\rangle<0$. Indeed, if $\|p\|=\|p-x\|$, then $a=b$ by the above, thus $x=-a e_{0}$ and therefore $\left\|e_{0} / 2\right\|_{M}=\left\|(a+1 / 2) e_{0}\right\|_{M}$. This implies $a \in\{0,-1\}$, hence $x \in\left\{0, e_{0}\right\}$, and therefore $e_{0}$ is a strict $M$-Voronoi vector, as announced.

Lemma 2.17. Let $D \in S_{2}^{++}$and let $\left(e_{0}, e_{1}, e_{2}\right)$ be a D-obtuse superbase. Let $M:=D^{-1}$ and $\left(v_{0}, v_{1}, v_{2}\right)=\left(e_{0}^{\perp}, e_{1}^{\perp}, e_{2}^{\perp}\right)$. Then $\left(v_{0}, v_{1}, v_{2}\right)$ is an $M$-obtuse superbase. In addition, for any $i \neq j$ one has $\left\langle e_{i}, D e_{j}\right\rangle<0$ iff $\left\langle v_{i}, M v_{j}\right\rangle<0$.

Proof. By construction one has $v_{0}, v_{1}, v_{2} \in \mathbb{Z}^{2}, v_{0}+v_{1}+v_{2}=\left(e_{0}+e_{1}+e_{2}\right)^{\perp}=0$, and $\operatorname{det}\left(v_{1}, v_{2}\right)=$ $\operatorname{det}\left(e_{1}, e_{2}\right)= \pm 1$. Thus $\left(v_{0}, v_{1}, v_{2}\right)$ is a superbase of $\mathbb{Z}^{2}$. On the other hand, the obtuseness properties come from the following identity: for any $e, e^{\prime} \in \mathbb{R}^{2}, D \in S_{2}^{++}$and $M:=D^{-1}$ one has

$$
\left\langle e^{\perp}, M e^{\perp}\right\rangle=\operatorname{det}(M)\left\langle e, D e^{\prime}\right\rangle .
$$

(In the special case $D=\mathrm{Id}$, this identity expresses that rotation by $\pi / 2$ is an isometry. In the general case $D=A^{T} A$ for some $A \in \mathrm{GL}_{2}(\mathbb{R})$, it follows from a linear change of variables and the relation $(A e)^{\perp}=\operatorname{cof}(A) e^{\perp}$ where $\operatorname{cof}(A)$ denotes the cofactor matrix.)

We are ready to prove Theorem 1.8, by adapting a result of [Mir16], devoted to operators without a first order term, and stated in terms of Voronoi vectors. 
Lemma 2.18 (Adapted from Theorem 1.3 in [Mir16]). Let $D \in S_{2}^{++}$, and let $D=\sum_{1 \leq i \leq I} \sigma_{i} e_{i} e_{i}^{\mathrm{T}}$ be the decomposition associated with a D-obtuse superbase by Lemma 2.3, pruned so that $\sigma_{i} \neq 0$ for all $1 \leq i \leq I$. Let also $D=\sum_{1 \leq i \leq I^{\prime}} \sigma_{i}^{\prime} e_{i}^{\prime} e_{i}^{\prime T}$ be another decomposition, with $I^{\prime}>0, \sigma_{i}^{\prime} \geq 0$, $e_{i}^{\prime} \in \mathbb{Z}^{2} \backslash\{0\}$ for all $1 \leq i \leq I^{\prime}$. Then

$$
\operatorname{Hull}\left\{ \pm e_{i} ; 1 \leq i \leq I\right\} \subseteq \operatorname{Hull}\left\{ \pm e_{i}^{\prime} ; 1 \leq i \leq I^{\prime}\right\}
$$

Proof. Theorem 1.3 in [Mir16] provides a similar statement, except that the vectors $\left( \pm e_{i}\right)_{1 \leq i \leq I}$ are defined as the strict $M$-Voronoi vectors, where $M=D^{-1}$. By Lemmas 2.16 and 2.17, the tensor decomposition here considered (2.4) is also supported on the set of strict $M$-Voronoi vectors, and the result follows.

Proof of Theorem 1.8. We use the notations of Theorem 1.8, and define $\sigma_{i}:=\rho_{i}+\rho_{-i}$ for all $1 \leq i \leq I$, and $\sigma_{i}^{\prime}:=\rho_{i}+\rho_{-i}^{\prime}$ for all $1 \leq i \leq I^{\prime}$. Note that $\sigma_{i}>0$ for all $1 \leq i \leq I$, since $\rho_{i} \neq 0$ or $\rho_{-i} \neq 0$ and both are non-negative. Then $D=\sum_{1<i<I} \sigma_{i} e_{i} e_{i}^{\mathrm{T}}=\sum_{1<i<I} \sigma_{i}^{\prime} e_{i}^{\prime} e_{i}^{\prime \mathrm{T}}$, and by Definition 1.5 the first decomposition comes from a $D$-obtuse superbase as in Lemma 2.3. Applying Lemma 2.18, and recalling that $e_{-i}:=-e_{i}$, we conclude the proof of Theorem 1.8.

\section{Proof of Theorem 1.6}

We establish in this section our main result, Theorem 1.6, on a compatibility relation needed for constructing our numerical scheme. This obstruction relates the grid scale $h$ (safely ignored in this section), with the first order term $\omega \in \mathbb{R}^{d}$ and the second order term $D \in S_{d}^{++}$of the discretized linear differential operator. More precisely, this result states that if $(\omega, D)$ is absolutely feasible (some discretization exists), then $\left(c_{d} \omega, D\right)$ is canonically feasible (our discretization exists), where $d \in\{2,3\}$ and $\left.\left.c_{d} \in\right] 0,1\right]$ is a constant.

The guiding principle of the proof is to adapt to the pair $(\omega, D) \in \mathbb{R}^{d} \times S_{d}^{++}$, of a vector and a symmetric positive definite matrix, the tools and techniques presented in $\S 2.1$ and $\S 2.2$, which originally apply to a matrix $D \in S_{d}^{++}$alone. The arguments are split into three parts, and proceed as follows. We define and describe in $\S 3.1$ a variant $\widetilde{\mathcal{M}}_{d} \subseteq \mathbb{R}^{d} \times S_{d}$ of Ryskov's polyhedron $\mathcal{M}_{d} \subseteq S_{d}$, see (2.5), involving an asymmetric perturbation of the constraints. The corresponding generalization $\widehat{\operatorname{Vor}}(\omega, D)$ of Voronoi's first reduction $\widetilde{\operatorname{Vor}}(D)$, see $(2.10)$, is discussed in $\S 3.2$. We conclude the proof of Theorem 1.6 in $\S 3.3$, by studying a low dimensional linear feasibility problem.

\subsection{A variant of Ryskov's polyhedron}

We study of a variant of Ryskov's polyhedron (2.5). Denoted $\widetilde{\mathcal{M}}_{d} \subseteq \mathbb{R}^{d} \times S_{d}$, it is defined as follows

$$
\widetilde{\mathcal{M}}_{d}:=\left\{(\eta, M) \in \mathbb{R}^{d} \times S_{d} ; \forall e \in \mathbb{Z}^{d} \backslash\{0\},\langle\eta, e\rangle+\langle e, M e\rangle \geq 1\right\} .
$$

This subsection is devoted to description of the vertices and edges of $\widetilde{\mathcal{M}}_{d}$, when $d \in\{2,3\}$, see Theorem 3.1 below (no other result from this section is used in the following ones). Surprisingly enough, this structure is only barely richer than that of Ryskov's original polyhedron, see Corollary 2.11, despite the higher dimension.

The concepts of superbase $b$ of $\mathbb{Z}^{d}$, the associated matrix $M_{b} \in S_{d}^{++}$, and the notion of adjacent superbases $\left(b, b^{\prime}\right)$, were introduced in Definitions 2.1, 2.7, and 2.9 respectively. Regular polyhedra and their edges are introduced in Definitions B.1 and B.2 of Appendix B.

Theorem 3.1. Let $d \in\{2,3\}$. Then $\widetilde{\mathcal{M}}_{d}$ is a regular polyhedron, with: 
(a) Vertices: $\left(0, M_{b}\right)$, for all superbases $b$ of $\mathbb{Z}^{d}$.

(b) Bounded edges: $\left[\left(0, M_{b}\right),\left(0, M_{b^{\prime}}\right)\right]$, for all adjacent superbases $b$ and $b^{\prime}$ of $\mathbb{Z}^{d}$.

(c) Unbounded edges: $\left\{\left(0, M_{b}\right)+\lambda\left(v_{I}, v_{I} v_{I}^{\mathrm{T}}\right) ; \lambda \geq 0\right\}$, for all superbases $b$ of $\mathbb{Z}^{d}$ and all $I \subsetneq$ $\{0, \cdots, d\}, I \neq \emptyset$, where $b=\left(v_{0}, \cdots, v_{d}\right)$ and $v_{I}:=\sum_{i \in I} v_{i}$.

The rest of this section is devoted to the proof of Theorem 3.1, following a line of arguments similar to the proof of Corollary 2.11. For commodity, we introduce a scalar product on $\mathbb{R}^{d} \times S_{d}$, as well as a family of elements $l_{e} \in \mathbb{R}^{d} \times S_{d}, e \in \mathbb{Z}^{d} \backslash\{0\}$, defined as follows:

$$
\langle(\eta, M),(\omega, D)\rangle:=\langle\eta, \omega\rangle+\operatorname{Tr}(M D), \quad l_{e}:=\left(e, e e^{\mathrm{T}}\right) .
$$

By construction $\left.\left\langle l_{e},(\eta, M)\right\rangle\right\rangle=\langle e, \eta\rangle+\langle e, M e\rangle$, which is convenient in view of (3.1). Observe that for any $\lambda_{1}, \cdots, \lambda_{I}, \mu_{1}, \cdots, \mu_{I} \in \mathbb{R}$ and $e_{1}, \cdots e_{I} \in \mathbb{Z}^{d}$, one has

$$
\sum_{1 \leq i \leq I} \frac{\lambda_{i}+\mu_{i}}{2}\left(e_{i}, e_{i} e_{i}^{\mathrm{T}}\right)+\frac{\lambda_{i}-\mu_{i}}{2}\left(-e_{i},\left(-e_{i}\right)\left(-e_{i}\right)^{\mathrm{T}}\right)=\left(\sum_{1 \leq i \leq I} \mu_{i} e_{i}, \sum_{1 \leq i \leq I} \lambda_{i} e_{i} e_{i}^{\mathrm{T}}\right) .
$$

Remark 3.2 (Erdahl's cone of quadratic functions). The set (3.1) is reminiscent of Erdahl's cone [Erd92, DSSV12], another inhomogeneous generalization of Voronoi's constructions, defined as follows:

$$
\mathcal{E}_{d}:=\left\{f \text { quadratic function on } \mathbb{R}^{d} ; \forall e \in \mathbb{Z}^{d}, f(e) \geq 0\right\}
$$

Recall that a quadratic function on is a map of the form $x \in \mathbb{R}^{d} \mapsto \alpha+\langle\eta, x\rangle+\langle x, M x\rangle$. Thus for any $f \in \mathcal{E}_{d}$, the normalized function $f / f(0)$ (assuming $f(0) \neq 0$ ) can be identified with an element of

$$
\left\{(\eta, M) \in \mathbb{R}^{d} \times S_{d} ; \forall e \in \mathbb{Z}^{d} \backslash\{0\},\langle\eta, e\rangle+\langle e, M e\rangle \geq-1\right\} .
$$

Despite the apparent similarity between (3.4) and (3.1), the set $\widetilde{\mathcal{M}}_{d}$ only resembles Erdahl's cone superficially. The set $\widetilde{\mathcal{M}}_{d}$ is more closely related with Ryskov's original polyhedron $\mathcal{M}_{d}$, as shown by Theorem 3.1 and Corollary 2.11.

Lemma 3.3. For all $(\eta, M) \in \widetilde{\mathcal{M}}_{d}$ one has $M \in \mathcal{M}_{d}$.

Proof. One has $\langle e, M e\rangle=\frac{1}{2}(\langle\eta, e\rangle+\langle e, M e\rangle)+\frac{1}{2}(\langle\eta,-e\rangle+\langle-e, M(-e)\rangle) \geq 1, \forall e \in \mathbb{Z}^{d} \backslash\{0\}$.

Lemma 3.4. The polyhedron $\widetilde{\mathcal{M}}_{d}$ is regular, in the sense of Definition B.1.

Proof. (i) Let $(\eta, M) \in \mathbb{R}^{d} \times S_{d}$ be such that $\|\eta\| \leq 1$ and $M \succeq 2$ Id. Then for any $e \in \mathbb{Z}^{d} \backslash\{0\}$ one has $\langle\eta, e\rangle+\langle e, M e\rangle \geq-\|e\|+2\|e\|^{2} \geq 1$ since $\|e\| \geq 1$. Thus $(\eta, M) \in \widetilde{\mathcal{M}}_{d}$, and therefore $\widetilde{\mathcal{M}}_{d}$ has a non-empty interior. (ii) Recalling that $\operatorname{Span}\left\{e e^{\mathrm{T}} ; e \in \mathbb{Z}^{d} \backslash\{0\}\right\}=S_{d}$, see Lemma 2.3, and using (3.3) one obtains $\operatorname{Span}\left\{\left(e, e e^{\mathrm{T}}\right) ; e \in \mathbb{Z}^{d} \backslash\{0\}\right\}=\mathbb{R}^{d} \times S_{d}$, as required. (iii) Let $(\eta, M) \in \widetilde{\mathcal{M}}_{d}$. Then $M \in \mathcal{M}_{d}$, by Lemma 3.3 , and therefore $M$ is a symmetric positive definite matrix, whose smallest eigenvalue is here denoted $\lambda_{\min }(M)>0$. Then for any $\left(\eta^{\prime}, M^{\prime}\right)$ such that $\left\|\eta-\eta^{\prime}\right\| \leq 1$ and $\left\|M-M^{\prime}\right\| \leq \lambda_{\min }(M) / 2$ one has for all $e \in \mathbb{Z}^{d} \backslash\{0\}$

$$
\begin{aligned}
\left\langle\eta^{\prime}, e\right\rangle+\left\langle e, M^{\prime} e\right\rangle & \geq-\left\|\eta^{\prime}\right\|\|e\|+\left(\lambda_{\min }(M)-\left\|M-M^{\prime}\right\|\right)\|e\|^{2} \\
& \geq\left(\lambda_{\min }(M)\|e\| / 2-\|\eta\|-1\right)\|e\| .,
\end{aligned}
$$

It follows that $\left\langle\eta^{\prime}, e\right\rangle+\left\langle e, M^{\prime} e\right\rangle \geq 2$ if $\|e\| \geq 2(\|\eta\|+3) / \lambda_{\min }(M)$. This shows that only finitely many of the constraints defining the polyhedron $\widetilde{\mathcal{M}}_{d}$ are active in the neighborhood of $(\eta, M) \in \widetilde{\mathcal{M}}_{d}$, as required. 
The next lemma describes a family of vertices of $\widetilde{\mathcal{M}}_{d}$.

Lemma 3.5. For any vertex $M$ of $\mathcal{M}_{d}$, the pair $(0, M)$ is a vertex of $\widetilde{\mathcal{M}}_{d}$. In addition, the active constraints at a vertex $M \in \mathcal{M}_{d}$, and at the corresponding vertex $(0, M) \in \widetilde{\mathcal{M}}_{d}$, are associated with the same vectors $e \in \mathbb{Z}^{d} \backslash\{0\}$.

Proof. We first check that $(0, M) \in \widetilde{\mathcal{M}}_{d}$. Indeed, for any $e \in \mathbb{Z}^{d} \backslash\{0\}$, one has $\left\langle l_{e},(0, M)\right\rangle=$ $\langle 0, e\rangle+\langle e, M e\rangle=\langle e, M e\rangle \geq 1$, since $M \in \mathcal{M}_{d}$.

We next prove that $(0, M)$ is a vertex of $\widetilde{\mathcal{M}}_{d}$, relying on the characterization of Remark B.3. By assumption, since $M$ is a vertex of $\mathcal{M}_{d}$, there exists $e_{1}, \cdots, e_{I}$ in $\mathbb{Z}^{d} \backslash\{0\}$ such that $\left\langle e_{i}, M e_{i}\right\rangle=1$ for all $1 \leq i \leq I$, and $\operatorname{Span}\left\{e_{i} e_{i}^{\mathrm{T}}\right\}_{1 \leq i \leq I}=S_{d}$. The latter property implies that $\left\{e_{i}\right\}_{i=1}^{I}$ spans $\mathbb{R}^{d}$, hence using (3.3) we obtain $\operatorname{Span}\left\{l_{e_{i}}\right\}_{1 \leq|i| \leq I}=\mathbb{R}^{d} \times S^{d}$, with the usual convention $e_{-i}=-e_{i}$. Since $\left\langle\left\langle l_{ \pm e_{i}},(0, M)\right\rangle=\left\langle e_{i}, M e_{i}\right\rangle=1\right.$, we conclude that $(0, M)$ is a vertex of $\widetilde{\mathcal{M}}_{d}$. The additional point is straightforward, since the vectors $e \in \mathbb{Z}^{d}$ associated to active constraints at $(0, M) \in \widetilde{\mathcal{M}}_{d}$ are characterized by the identity $1=\langle e, 0\rangle+\langle e, M e\rangle=\langle e, M e\rangle$.

It the rest of this section, we compute the edges emanating from a vertex $(0, M) \in \widetilde{\mathcal{M}}_{d}$ in the form of Lemma 3.5. We apply the strategy of $\S B .3$ to compute the outgoing direction of each edge, and eventually only encounter the two following cases:

(i) The computed edge direction has the form $\nu=(0, N)$ for some $N \in S_{d}$, hence the corresponding edge is internal to $\widetilde{\mathcal{M}}_{d} \cap\left(\{0\} \times S_{d}\right)=\{0\} \times \mathcal{M}_{d}$. Since the edges of $\mathcal{M}_{d}$ are known, see Corollary 2.11, this must be a bounded edge in the form of Theorem 3.1 (b).

(ii) The computed edge direction has the form $\nu=\left(v, v v^{\mathrm{T}}\right.$ ), where $v \in \mathbb{Z}^{d}$ (more precisely, $v$ has the form indicated in Theorem 3.1 (c)). Thus for any $e$ in $\mathbb{Z}^{d} \backslash\{0\}$,

$$
\left\langle\left\langle l_{e}, \nu\right\rangle\right\rangle=\left\langle\left\langle\left(e, e e^{\mathrm{T}}\right),\left(v, v v^{\mathrm{T}}\right)\right\rangle=\langle e, v\rangle+\operatorname{Tr}\left(e e^{\mathrm{T}} v v^{\mathrm{T}}\right)=\langle e, v\rangle+\langle e, v\rangle^{2} .\right.
$$

Since $e$ and $v$ have integer coordinates, the scalar product $\langle e, v\rangle$ is an integer, and therefore $\left\langle l_{e}, \nu\right\rangle \geq 0$ (with equality iff $\langle e, v\rangle \in\{0,-1\}$ ). Thus $\nu$ yields an unbounded edge in $\widetilde{\mathcal{M}}_{d}$ starting from $(0, M)$, in the form of Theorem 3.1 (c).

The graph defined by the edges and vertices of a regular polyhedron is connected, see Appendix B. Once the above dichotomy is established, it follows that $\widetilde{\mathcal{M}}_{d}$ has no other vertices than those already found in Lemma 3.5, which concludes the proof of Theorem 3.1.

\section{Notation (i-ii) and (A-D) in §3.1.1 and 3.1.2.}

We establish the above dichotomy (i-ii) in $\S 3.1 .1$ and 3.1.2, in dimension two and three respectively. For that purpose, we rely on the algorithm presented in $\S$ B.3 for enumerating the outgoing edges from a vertex in a polyhedron, and explicitly refer to its steps (A-D).

\subsubsection{Edges of $\widetilde{\mathcal{M}}_{2}$}

Let $b=\left(v_{0}, v_{1}, v_{2}\right)$ be a superbase of $\mathbb{Z}^{2}$, and let $M_{b}$ be the corresponding vertex of $\mathcal{M}_{2}$, see (2.6). By Lemma 2.8, the active constraints at the vertex $M_{b} \in \mathcal{M}_{2}$ correspond to the set of vectors $E:=\left\{e_{i j} ; i, j \in\{0,1,2\}, i \neq j\right\}$ associated with the superbase $b$, see Definition 2.2. By Lemma $3.5,\left(0, M_{b}\right)$ is a vertex of the polyhedron $\widetilde{\mathcal{M}}_{2}$, at which the constraints associated with the same vectors $e \in E$ are active. Since the number $\#(E)=6$ of active constraints at $\left(0, M_{b}\right) \in \widetilde{\mathcal{M}}_{2}$ exceeds the dimension $\operatorname{dim}\left(\mathbb{R}^{2} \times S_{2}\right)=2+3=5$ of the embedding vector space, 
the vertex is degenerate. The edges containing $\left(0, M_{b}\right) \in \widetilde{\mathcal{M}}_{2}$ are obtained by selecting 4 out of the six active constraints, in other words by removing two elements from the set $E$. The following cases can be distinguished:

- Removing $e_{12}$ and $e_{21}$. The corresponding direction is $\nu=\left(0, v_{1} \otimes v_{2}\right)$, which lies within $\{0\} \times S_{d}$, and thus falls in case (i). Validation of the direction: one has $\left\langle l_{e_{01}}, \nu\right\rangle=$ $\left\langle e_{01}, v_{1} \otimes v_{2} e_{01}\right\rangle=\left\langle e_{01}, v_{1}\right\rangle\left\langle e_{01}, v_{2}\right\rangle=0$, since $\left\langle e_{01}, v_{2}\right\rangle=0$. Likewise $\left.\left\langle l_{e}, \nu\right\rangle\right\rangle=0$ for all $e \in\left\{ \pm e_{01}, \pm e_{02}\right\}$, hence $\nu$ obeys the conditions of (B) of Algorithm $\S \mathrm{B} .3$.

- Removing $e_{01}$ and $e_{02}$. The corresponding direction is $\nu=\left(v_{0}, v_{0} \otimes v_{0}\right)$, which falls in the case (ii) of an unbounded edge. Validation of the direction: one has $\left.\left\langle l_{e_{12}}, \nu\right\rangle\right\rangle=$ $\left\langle e_{12}, v_{0}\right\rangle^{2}+\left\langle e_{12}, v_{0}\right\rangle=0^{2}+0=0$, and $\left\langle\left\langle l_{e_{10}}, \nu\right\rangle=\left\langle e_{10}, v_{0}\right\rangle^{2}+\left\langle e_{10}, v_{0}\right\rangle=(-1)^{2}+(-1)=0\right.$. Likewise for $e \in\left\{e_{21}, e_{20}\right\}$.

- Removing $e_{01}$ and $e_{20}$. The corresponding direction is $\nu=\left(v_{0}, v_{1} \otimes v_{1}-v_{2} \otimes v_{2}\right)$, but it does not correspond to an edge, since it is eliminated in step (C) of Algorithm §B.3. Indeed, noting that $\left\langle l_{e}, \nu\right\rangle=\left\langle e, v_{1}\right\rangle^{2}-\left\langle e, v_{2}\right\rangle^{2}+\left\langle e, v_{0}\right\rangle$ we obtain

$$
\begin{aligned}
\left\langle l_{e_{10}}, \nu\right\rangle & =1^{2}-0^{2}-1=0, \\
\left\langle l_{e_{02}}, \nu\right\rangle & =0^{2}-(-1)^{2}+1=0, \\
\left\langle l_{ \pm e_{12}}, \nu\right\rangle & =( \pm 1)^{2}-(\mp 1)^{2}+0,
\end{aligned}
$$

showing that the direction $\nu$ is correct. However since

$$
\left\langle l_{e_{01}}, \nu\right\rangle=(-1)^{2}-0^{2}+1=2, \quad\left\langle l_{e_{20}}, \nu\right\rangle=0^{2}-1^{2}-1=-2,
$$

have opposite signs, the direction $\nu$ does not yield an edge of positive length.

There are 15 distinct two element subsets of $E:=\left\{e_{i j} ; i, j \in\{0,1,2\}, i \neq j\right\}$, and we have considered just three. However by permuting indices, the above considered cases respectively cover 3,6 , and again 6 , distinct two element subsets $E$. Thus our enumeration is complete, and Theorem 3.1 is proved in dimension $d=2$.

\subsubsection{Edges of $\widetilde{\mathcal{M}}_{3}$}

Let $b=\left(v_{0}, v_{1}, v_{2}, v_{3}\right)$ be a superbase of $\mathbb{Z}^{3}$, and let $M_{b}$ be the corresponding vertex of $\mathcal{M}_{3}$, see (2.6). By Lemma 2.8, the active constraints at the vertex $M_{b} \in \mathcal{M}_{3}$ correspond to the set of vectors $E:=\left\{e_{i j} ; i, j \in\{0,1,2,3\}, i \neq j\right\}$ associated with the superbase $b$, see Definition 2.2. By Lemma $3.5,\left(0, M_{b}\right)$ is a vertex of the polyhedron $\widetilde{\mathcal{M}}_{3}$, at which the constraints associated with the same vectors $e \in E$ are active. Since the number $\#(E)=12$ of active constraints at $\left(0, M_{b}\right) \in \widetilde{\mathcal{M}}_{2}$ exceeds the dimension $\operatorname{dim}\left(\mathbb{R}^{3} \times S_{3}\right)=3+6=9$ of the embedding vector space, the vertex is degenerate. The edges containing $\left(0, M_{b}\right) \in \widetilde{\mathcal{M}}_{3}$ are obtained by selecting 8 out of the twelve active constraints, in other words by removing four elements from the set $E$. The following cases can be distinguished:

- Removing $\pm e_{01}$ and two other unspecified elements of $E$. If the subset is not rejected in step (B), then the corresponding direction is $\nu=\left(0, v_{0} \otimes v_{1}\right)$, which lies within $\{0\} \times S_{d}$ and thus falls into case (i). Validation of the direction: one has $\left\langle l_{e_{i j}}, \nu\right\rangle=\left\langle e_{i j}, v_{0}\right\rangle\left\langle e_{i j}, v_{1}\right\rangle=0$ as soon as $\{i, j\} \neq\{0,1\}$, hence $\nu$ obeys the conditions of $(\mathrm{B})$. 
- Removing $\alpha_{01} e_{01}, \alpha_{02} e_{02}, \alpha_{03} e_{03}$ and another unspecified element of $E$, where $\alpha_{01}, \alpha_{02}, \alpha_{03} \in$ $\{-1,1\}$. The corresponding direction is, up to a global sign change,

$$
\nu=\left(-v_{0}, \alpha_{01} v_{0} \otimes v_{1}+\alpha_{02} v_{0} \otimes v_{2}+\alpha_{03} v_{0} \otimes v_{3}\right) .
$$

It is rejected in step $(\mathrm{B})$ or $(\mathrm{C})$, unless $\alpha_{01}=\alpha_{02}=\alpha_{03}$ in which case $\nu=\left(-\alpha_{01} v_{0}, v_{0} \otimes v_{0}\right)$ (here with the correct sign) falls in case (ii) and defines an unbounded edge. (Note that $v_{0} \otimes v_{1}+v_{0} \otimes v_{2}+v_{0} \otimes v_{3}=-v_{0} \otimes v_{0}$ since $v_{1}+v_{2}+v_{3}=-v_{0}$.) Indeed, for any $i, j \in\{1,2,3\}$ such that $i \neq j$ one computes

$$
\left\langle l_{ \pm e_{0 i}}, \nu\right\rangle=-\alpha_{0 i} \mp 1, \quad\left\langle l_{e_{i j}}, \nu\right\rangle=0 .
$$

- Removing $\alpha_{01} e_{01},-\alpha_{12} e_{12}, \alpha_{23} e_{23},-\alpha_{30} e_{30}$, where $\alpha_{01}, \alpha_{12}, \alpha_{23}, \alpha_{30}$ belong to $\{-1,1\}$. Then the corresponding direction is, up to a global sign change,

$$
\nu=\left(v_{1}+v_{3}, \alpha_{01} v_{0} \otimes v_{1}+\alpha_{12} v_{1} \otimes v_{2}+\alpha_{23} v_{2} \otimes v_{3}+\alpha_{30} v_{3} \otimes v_{0}\right) .
$$

It is rejected in step $C$, unless $\alpha_{01}=\alpha_{12}=\alpha_{23}=\alpha_{30}$, in which case $\nu=(v, v \otimes v)$ (here with the correct sign) with $v=-\alpha_{01}\left(v_{1}+v_{3}\right)=\alpha_{01}\left(v_{0}+v_{2}\right)$ falls in case (ii) and thus defines an unbounded edge. (Note that $-\left(v_{1}+v_{3}\right) \otimes\left(v_{1}+v_{3}\right)=\left(v_{0}+v_{2}\right) \otimes\left(v_{1}+v_{3}\right)=$ $v_{0} \otimes v_{1}+v_{1} \otimes v_{2}+v_{2} \otimes v_{3}+v_{3} \otimes v_{0}$ since $v_{0}+v_{2}=-\left(v_{1}+v_{3}\right)$.) Indeed, we check that

$$
\begin{aligned}
\left\langle l_{e_{02}}, \nu\right\rangle & =0+0+0+0+0, \quad \text { likewise for } e \in\left\{ \pm e_{02}, \pm e_{13}\right\} \\
\left\langle l_{ \pm e_{01}}, \nu\right\rangle & =-\alpha_{01} \pm 1, \quad \text { likewise for } e \in\left\{ \pm e_{01}, \pm e_{12}, \pm e_{23}, \pm e_{30}\right\}
\end{aligned}
$$

Finally, we need to show that all the possible 4 element subsets $S \subseteq\left\{e_{i j} ; i \neq j\right\}$ correspond to one of the considered cases, up to a permutation of the superbase. We refer to $i$ and $j$ as the indices of a vector $e_{i j} \in S$. If two elements of $S$ share the same two indices, a.k.a. $e_{i j}, e_{j i} \in S$ for some $i \neq j$, then we fall in the first case. Otherwise, if (at least) three elements of $S$ share one index, then we fall in the second case. Otherwise, each index $i \in\{0, \cdots, 3\}$ appears in at most two elements of $S$, thus exactly two since $\#(S)=4=\#\{0, \cdots, 3\}$. It follows that the indices of $S$ define a cycle, and we fall in the last case.

\subsection{A variant of Voronoi's first reduction}

We introduce and study a variant of Voronoi's first reduction, applying to pairs $(\omega, D)$ of a vector $\omega \in \mathbb{R}^{d}$ and a positive definite symmetric tensor $D \in S_{d}^{++}$, instead of the matrix $D$ alone in the original formulation (2.10). It is defined as follows:

$$
\widetilde{\operatorname{Vor}}(\omega, D):=\inf \left\{\langle\omega, \eta\rangle+\operatorname{Tr}(D M) ;(\eta, M) \in \widetilde{\mathcal{M}}_{d}\right\}
$$

Somewhat surprisingly, this generalization of Voronoi's first reduction reduces to the original one, subject to a compatibility condition.

Theorem 3.6. Let $d \leq 3$. For any $(D, \omega) \in S_{d}^{++} \times \mathbb{R}^{d}$ one has

$$
\widetilde{\operatorname{Vor}}(D, \omega)= \begin{cases}-\infty & \text { if } \exists v \in \mathbb{Z}^{d} \backslash\{0\},\langle v, D v\rangle+\langle\omega, v\rangle<0, \\ \operatorname{Vor}(D) & \text { otherwise. }\end{cases}
$$


Proof. The result follows from the description of the vertices and unbounded edges of the polytope $\widetilde{\mathcal{M}}_{d}$ in Theorem 3.1, and from the general expression (B.2) of the value of a linear program. Note also that any $v_{1} \in \mathbb{Z}^{d} \backslash\{0\}$ with co-prime coordinates can be completed into a basis $v_{1}, \cdots, v_{d}$ of $\mathbb{Z}$, hence also into a superbase with $v_{0}:=-\left(v_{1}+\cdots+v_{d}\right)$. Hence the set of directions of all unbounded edges of $\widetilde{\mathcal{M}}_{d}$, see Theorem 3.1 (c), is $\mathbb{Z}^{d} \backslash\{0\}$.

Proposition 3.7. Let $d \leq 3$, and let $(\omega, D) \in \mathbb{R}^{d} \times S_{d}^{++}$. The following are equivalent:

(i) The pair $(\omega, D)$ is absolutely feasible.

(ii) The linear program $\widetilde{\operatorname{Vor}}(\omega, D)$ is bounded.

In case (ii), any set of KKT relations for $\widetilde{\operatorname{Vor}}(\omega, D)$ yields a simultaneous decomposition of $(\omega, D)$, showing (i) explicitly.

Proof. Proof that (i) $\Rightarrow$ (ii). Assume that $(\omega, D)$ is absolutely feasible, and denote by $\rho_{i} \geq 0$ the weights, and $e_{i} \in \mathbb{Z}^{d}$ the offsets of the corresponding decomposition, so that

$$
\omega=\sum_{1 \leq i \leq I} \rho_{i} e_{i}, \quad D=\sum_{1 \leq i \leq I} \rho_{i} e_{i} e_{i}^{T} .
$$

Then for any $(M, \eta) \in \widetilde{\mathcal{M}}_{d}$, one obtains using the identity $\langle e, M e\rangle=\operatorname{Tr}\left(M e e^{\mathrm{T}}\right)$

$$
\langle\omega, \eta\rangle+\operatorname{Tr}(D M)=\sum_{1 \leq i \leq I} \rho_{i}\left(\left\langle\omega, e_{i}\right\rangle+\left\langle e_{i}, M e_{i}\right\rangle\right) \geq \sum_{1 \leq i \leq I} \rho_{i} \geq 0
$$

Therefore $\widetilde{\operatorname{Vor}}(D, \omega) \geq 0>-\infty$ is bounded.

Proof that (ii) $\Rightarrow$ (i). By Proposition 2.12 there exists a vertex $M_{b}$ of $\mathcal{M}_{d}$, where $b$ is a superbase of $\mathbb{Z}^{d}$, such that $\operatorname{Vor}(D)=\operatorname{Tr}\left(D M_{b}\right)$. By Lemmas 2.8 and $3.5,\left(0, M_{b}\right)$ is a vertex of $\widetilde{\mathcal{M}}_{d}$ at which finitely many constraints $\left(e_{i}\right)_{i=1}^{I}$ are active. By Theorem 3.6, $\widetilde{\operatorname{Vor}}(D)=$ $\operatorname{Vor}(D)=\operatorname{Tr}\left(D M_{b}\right)=\langle\omega, 0\rangle+\operatorname{Tr}\left(D M_{b}\right)$ and this minimum is attained at the vertex $\left(0, M_{b}\right)$. The KKT relations express that there exists non-negative weights $\left(\rho_{i}\right)_{i=1}^{I}$ (possibly non-unique) such that the objective function and the weighted sum of the constraints are equal: one has $\langle\omega, \eta\rangle+\operatorname{Tr}(D M)=\sum_{1 \leq i \leq I} \rho_{i}\left(\left\langle e_{i}, \eta\right\rangle+\left\langle e_{i}, M e_{i}\right\rangle\right)$ for all $(\eta, M) \in \mathbb{R}^{d} \times S_{d}$. From this point, the simultaneous decomposition (3.7) follows by identification, as announced.

Remark 3.8 (Degeneracy of the vertices of $\widetilde{\mathcal{M}}_{d}$ ). The vertices of $\widetilde{\mathcal{M}}_{d}$ are degenerate, in dimension $d \in\{2,3\}$, in the sense that exactly $d(d+1)$ constraints are active, which is strictly greater than $\operatorname{dim}\left(\mathbb{R}^{d} \times S_{d}\right)=d(d+1) / 2+d$. As a result, the KKT relations for the linear $\operatorname{program} \operatorname{Vor}(\omega, D)$ in general do not uniquely determine the decomposition (3.7) of the pair $(\omega, D)$. This is in constrast with Voronoi's first reduction in dimension $d \leq 3$, see Remark 2.13.

\subsection{Local study of feasibility}

In this section, we compare the conditions of canonical and absolute feasibility of a pair $(\omega, D)$, in dimension $d \leq 3$, concluding the proof of Theorem 1.6. For that purpose, we fix a symmetric positive definite matrix $D \in S_{d}^{++}$, denote by $b=\left(v_{0}, \cdots, v_{d}\right)$ a $D$-obtuse superbase, and recall Selling's decomposition (2.4)

$$
D=\sum_{0 \leq i<j \leq d} \sigma_{i j} e_{i j} e_{i j}^{\mathrm{T}}
$$


where $\sigma_{i j}:=-\left\langle v_{i}, D v_{j}\right\rangle \geq 0$ and where $e_{i j} \in \mathbb{Z}^{d} \backslash\{0\}$ for all $0 \leq i<j \leq d$ is introduced in Definition 2.2. In this subsection, for notational convenience, the indices $i$ and $j$, are always implicitly constrained to lie in the set $\{0, \cdots, d\}$.

We characterize, in the next proposition, the canonical and absolute feasibility of a pair $(\omega, D)$ in terms of Selling's decomposition of $D$. The argument, in the case of absolute feasibility, heavily relies on the results established in $§ 3.2$. An interesting byproduct is that, if a pair $(\omega, D)$ is absolutely feasible, then it admits a decomposition (1.3) whose offsets are those of Selling's formula (3.8) for $D$ (and the opposite offsets).

Proposition 3.9. Assume $d \leq 3$. Let $\omega \in \mathbb{R}^{d}$ and $D \in S_{d}^{++}$. We use the notations $b$ and $\left(\sigma_{i j}, e_{i j}\right)_{i<j}$ of Selling's decomposition (3.8). Then

- $(\omega, D)$ is absolutely feasible iff there exists $\mu_{i j} \in[-1,1]$, for all $0 \leq i<j \leq d$, such that $\omega=\sum_{i<j} \mu_{i j} \sigma_{i j} e_{i j}$.

- $(\omega, D)$ is canonically feasible iff $\left|\left\langle e_{i j}, D^{-1} \omega\right\rangle\right| \leq 1$ for all $0 \leq i<j \leq d$ such that $\sigma_{i j}>0$.

Proof. First equivalence. If the pair $(\omega, D)$ is absolutely feasible, then by Proposition 3.7 the linear program $\widetilde{\operatorname{Vor}}(\omega, D)$ is bounded, and attains its minimum at the vertex $\left(0, M_{b}\right)$, at which the active constraints are associated with the vectors $e_{i j}, i \neq j$. By the KKT relations, there exists non-negative weights $\rho_{i j}, i \neq j$, such that

$$
\omega=\sum_{i \neq j} \rho_{i j} e_{i j} \quad D=\sum_{i \neq j} \rho_{i j} e_{i j} e_{i j}^{T} .
$$

Recalling that $e_{j i}=-e_{i j}$ for all $i \neq j$, we obtain

$$
\omega=\sum_{i<j}\left(\rho_{i j}-\rho_{j i}\right) e_{i j} \quad D=\sum_{i<j}\left(\rho_{i j}+\rho_{j i}\right) e_{i j} e_{i j}^{T} .
$$

By uniqueness of Selling's decomposition, see Remark 2.13, one has $\sigma_{i j}=\rho_{i j}+\rho_{j i}$ for all $i<j$. Denoting $\mu_{i j}:=\left(\rho_{i j}-\rho_{j i}\right) / \sigma_{i j} \in[-1,1]$ when $\sigma_{i j}>0$ (and e.g. $\mu_{i j}=0$ if $\sigma_{i j}=0$ ), we obtain $\omega=\sum_{i<j} \mu_{i j} \sigma_{i j} e_{i j}$ as announced. The reverse implication is trivial, by defining $\rho_{i j}=\sigma_{i j}\left(1+\mu_{i j}\right) / 2$ and $\rho_{j i}=\sigma_{i j}\left(1-\mu_{i j}\right) / 2$, for all $i<j$.

Second equivalence. By construction, see Definition 1.5, the pair $(\omega, D)$ obeys is canonically feasible iff $\sigma_{i j}\left(1+\varepsilon\left\langle e_{i j}, \eta\right\rangle\right) \geq 0$ for all $i<j$ and all $\varepsilon \in\{-1,1\}$, where $\eta:=D^{-1} \omega$. This is indeed equivalent to $\left|\left\langle e_{i j}, D^{-1} \omega\right\rangle\right| \leq 1$, for all $i<j$ such that $\sigma_{i j}>0$, as announced.

We next state two technical lemmas which, combined with Proposition 3.9 above, let us conclude the proof of Theorem 1.6. The proof of Lemma 3.10 is postponed to §3.3.1.

Lemma 3.10. Let $D \in S_{d}^{++}, d \leq 3$. We use the notations $b,\left(\sigma_{i j}, e_{i j}\right)_{i<j}$ of Selling's decomposition (3.8). Then $\left|\left\langle e_{i j}, M e_{k l}\right\rangle\right| \leq\left\|e_{i j}\right\|_{M}^{2}$ for all $i<j$ and all $k<l$, where $M:=D^{-1}$.

Lemma 3.11. Let $D=\sum_{r=1}^{R} \sigma_{r} e_{r} e_{r}^{T}$, where $\sigma_{r} \geq 0, e_{r} \in \mathbb{R}^{d}$ for all $1 \leq r \leq R$, and $R$ is a positive integer. If $D$ is positive definite, then $\sigma_{r}\left\langle e_{r}, D^{-1} e_{r}\right\rangle \leq 1$ for all $1 \leq r \leq R$.

Proof. For any $1 \leq r \leq R$, one has $D \succeq \sigma_{r} e_{r} e_{r}^{\mathrm{T}}$, in the sense of symmetric matrices. Therefore, letting $v_{r}:=D^{-1} e_{r}$, we obtain $\left\langle e_{r}, D^{-1} e_{r}\right\rangle=\left\langle v_{r}, D v_{r}\right\rangle \geq \sigma_{r}\left\langle v_{r}, e_{r}\right\rangle^{2}=\sigma_{r}\left\langle e_{r}, D^{-1} e_{r}\right\rangle^{2}$. This implies $1 \geq \sigma_{r}\left\langle e_{r}, D^{-1} e_{r}\right\rangle$, as announced. 
Proof of Theorem 1.6. Assume that $(D, \omega)$ is absolutely feasible. Then for any $i<j$, using the notations of Proposition 3.9, we obtain

$$
\begin{aligned}
\left|\left\langle e_{i j}, D^{-1} \omega\right\rangle\right| & \leq \sum_{k<l} \sigma_{k l}\left|\mu_{k l}\right|\left|\left\langle e_{i j}, D^{-1} e_{k l}\right\rangle\right| \\
& \leq \sum_{k<l} \sigma_{k l}\left\langle e_{k l}, D^{-1} e_{k l}\right\rangle \\
& \leq \sum_{k<l} 1=d(d+1) / 2 .
\end{aligned}
$$

The three inequalities follows, successively, from Proposition 3.9 (first point), Lemma 3.10, and Lemma 3.11. If follows from Proposition 3.9 (second point) that $(D, \omega / C)$ is canonically feasible, with $C:=d(d+1) / 2$, as announced.

\subsubsection{Proof of Lemma 3.10}

Throughout this subsection, we use for convenience the notation $\langle v, w\rangle_{M}:=\langle v, M w\rangle$, for any $v, w \in \mathbb{R}^{d}, M \in S_{d}^{++}$. We use the notations of Lemma 3.10. In particular $D \in S_{d}^{++}, M:=D^{-1}$, $b=\left(v_{0}, \cdots, v_{d}\right)$ is a $D$-obtuse superbase, and $\left(\sigma_{i j}, e_{i j}\right)_{i<j}$ are the coefficients and offsets of Selling's decomposition (3.8) of $D$. As before, the indices $i, j$ implicitly lie in $\{0, \cdots, d\}$.

Proof in dimension $d=2$. Assume that the superbase $b=\left(v_{0}, v_{1}, v_{2}\right)$ satisfies

$$
\operatorname{det}\left(v_{1}, v_{2}\right)=1 \text {, }
$$

without loss of generality and up to exchanging $v_{1}$ and $v_{2}$. Then $\left(e_{12}, e_{20}, e_{01}\right)=\left(v_{0}^{\perp}, v_{1}^{\perp}, v_{2}^{\perp}\right)$ by (2.2), and this triplet is an $M$-obtuse superbase by Lemma 2.17. Denoting $\left(w_{0}, w_{1}, w_{2}\right):=$ $\left(e_{12}, e_{20}, e_{01}\right)$ one obtains

$$
-\left\langle w_{0}, w_{1}\right\rangle_{M}-\left\langle w_{0}, w_{2}\right\rangle_{M}=\left\langle w_{0},-w_{1}-w_{2}\right\rangle_{M}=\left\|w_{0}\right\|_{M}^{2},
$$

and therefore $0 \leq-\left\langle w_{0}, w_{1}\right\rangle_{M} \leq\left\|w_{0}\right\|_{M}^{2}$. Likewise $0 \leq-\left\langle w_{i}, w_{j}\right\rangle_{M} \leq\left\|w_{i}\right\|_{M}^{2}$ for all $i \neq j$, which is the announced result.

Proof in dimension $d=3$. Denote $w_{i j}:=v_{i} \times v_{j}$ for all $i \neq j$. In the following, $\{i, j, k, l\}$ denotes an arbitrary permutation of $\{0,1,2,3\}$, thus for instance $w_{i j}= \pm e_{k l}$ by $(2.2)$. Note also that

$$
w_{i j}=-w_{j i}, \quad \text { and } \quad w_{i j}+w_{i k}+w_{i l}=v_{i} \times\left(v_{j}+v_{k}+v_{l}\right)=-v_{i} \times v_{i}=0 .
$$

The scalar products defined by $D \in S_{3}^{++}$and its inverse $M:=D^{-1}$ are related by the following identity, where $u, v, w \in \mathbb{R}^{3}$

$$
\operatorname{det}(D)\langle u \times v, u \times w\rangle_{M}=\|u\|_{D}^{2}\langle v, w\rangle_{D}-\langle u, v\rangle_{D}\langle u, w\rangle_{D} .
$$

(In the case $D=\mathrm{Id}$ this is known as the Binet-Cauchy identity. In the general case where $D=A^{T} A$ for some $A \in \mathrm{GL}_{3}(\mathbb{R})$ it follows from a linear change of variables and the relation $(A u) \times(A v)=\operatorname{cof}(A)(u \times v)$ where $\operatorname{cof}(A)$ denotes the cofactor matrix. $)$

Choosing $u=v_{i}, v=v_{j}$ and $w=v_{k}$, we obtain that $\left\langle w_{i j}, w_{i k}\right\rangle_{M} \leq 0$. On the other hand

$$
\begin{aligned}
-\left\langle w_{i j}, w_{i k}\right\rangle_{M}-\left\langle w_{i j}, w_{i l}\right\rangle_{M} & =\left\langle w_{i j}, v_{i} \times\left(-v_{k}-v_{l}\right)\right\rangle_{M} \\
& =\left\langle w_{i j}, v_{i} \times\left(v_{i}+v_{j}\right)\right\rangle_{M} \\
& =\left\|w_{i j}\right\|_{M}^{2},
\end{aligned}
$$


thus $0 \leq-\left\langle w_{i j}, w_{i k}\right\rangle_{M} \leq\left\|w_{i j}\right\|_{M}^{2}$. Finally, since $-w_{k l}=w_{k i}+w_{k j}$, we obtain that

$$
-\left\langle w_{i j}, w_{k l}\right\rangle_{M}=\left\langle w_{i j}, w_{k i}+w_{k j}\right\rangle_{M}=-\left\langle w_{i j}, w_{i k}\right\rangle_{M}+\left\langle w_{j i}, w_{j k}\right\rangle_{M}
$$

and therefore, by the previous estimate, $-\left\|w_{i j}\right\|_{M}^{2} \leq\left\langle w_{i j}, w_{k l}\right\rangle_{M} \leq\left\|w_{i j}\right\|_{M}^{2}$. This concludes the proof of Lemma 3.10.

\section{Numerical experiments}

We illustrate the PDE discretization introduced in this paper with synthetic numerical experiments, in dimension $d \in\{2,3\}$, involving linear and quasi-linear operators, and using Dirichlet boundary conditions on a non-square and non-smooth domain. Let us mention that a close variant of the proposed scheme, involving the divergence form operator $\operatorname{div}(D(x)(\nabla u(x)-\omega(x) u(x)))$ featuring both a first and second order term, is used in $\left[\mathrm{PCC}^{+} 19\right]$ for image impainting purposes in dimension $d=2$, in collaboration with one of the authors. See also [FM14] for applications to image denoising in dimension $d \in\{2,3\}$, with an operator lacking the first order term however. Additional concrete applications of the proposed scheme will be the object of future work.

The PDEs addressed numerically in this section take the form

$$
\mathcal{L} u(x)=f(x), \forall x \in \Omega, \quad u(x)=g(x), \forall x \in \partial \Omega,
$$

where $\left.\Omega:=\left\{x \in \mathbb{R}^{d} ;\|x\|<1\right\} \cup\right] 0,1^{d}$ is the union of the $d$-dimensional unit ball and of the $d$-dimensional unit cube. The PDE operator $-\mathcal{L} u(x)$ is chosen as the following linear (resp. quasi-linear) expression

$$
\langle\omega(x), \nabla u(x)\rangle+\frac{1}{2} \operatorname{Tr}\left(D(x) \nabla^{2} u(x)\right) \quad\left(\operatorname{resp} . \frac{1}{2}\langle\omega(x), \nabla u(x)\rangle^{2}+\frac{1}{2} \operatorname{Tr}\left(D(x) \nabla^{2} u(x)\right)\right)
$$

whose coefficients $\omega: \bar{\Omega} \rightarrow \mathbb{R}^{d}$ and $D: \bar{\Omega} \rightarrow S_{d}^{++}$are defined for any $x=\left(x_{1}, \cdots, x_{d}\right)$ in $\mathbb{R}^{d}$ by

$$
\begin{aligned}
\omega(x) & :=\frac{2-\cos \left(\pi x_{1}\right)}{3} \omega_{0}(x), \\
D(x) & :=\mu \frac{2+\cos \left(\pi x_{1}\right)}{3}\left(\nu I_{d}+(1-\nu) \omega_{0}(x / 2) \omega_{0}(x / 2)^{T}\right),
\end{aligned}
$$

where the parameters $\mu, \nu>0$ are specified in Figures 3 to 6 , and where $\omega_{0}$ is the field of unit vectors defined by

$$
\omega_{0}(x):= \begin{cases}\left(\cos \left(\pi x_{2}\right), \sin \left(\pi x_{2}\right)\right) & \text { if } d=2 \\ \left(\cos \left(\pi x_{2}\right), \sin \left(\pi x_{2}\right) \cos \left(\pi x_{3}\right), \sin \left(\pi x_{2}\right) \sin \left(\pi x_{3}\right)\right) & \text { if } d=3\end{cases}
$$

This particular choice of operator and coefficients is only meant to be reasonably simple and explicit, and to feature substantial anisotropy for the second order term. It also allows for a direct analytic verification of the assumptions of Theorem 1.7 ensuring the DDE property, see the last paragraph of this section.

By construction, the condition number $\operatorname{Cond}(D):=\sqrt{\|D\|\left\|D^{-1}\right\|}$ of the symmetric matrices involved in the PDE operators (4.2) is $\operatorname{Cond}(D(x))=\nu^{-\frac{1}{2}}$. In the experiments we use $\nu=1 / 10$, so that $\operatorname{Cond}(D(x))=\sqrt{10} \approx 3.1$, and the radius of the numerical scheme stencil never exceeds $\sqrt{5}$ (times the discretization grid scale $h$ ) both in dimension two and three. Stronger anisotropies, up to $\operatorname{Cond}(D(x))=10$, are routinely used in the applications of numerical schemes based on 


\begin{tabular}{c|c|c|c} 
Stencil radius & $d=2$ & $d=3$ & $d=3, \lambda_{1}=\lambda_{2} \leq \lambda_{3}$ \\
\hline $\operatorname{Cond}(D) \leq \sqrt{10}$ & $\sqrt{5} \approx 2.2$ & $\sqrt{11} \approx 3.3$ & $\sqrt{5} \approx 2.2$ \\
$\operatorname{Cond}(D) \leq 10$ & $\sqrt{26} \approx 5.1$ & $\sqrt{69} \approx 8.3$ & $\sqrt{30} \approx 5.5$
\end{tabular}

Table 1: Maximum stencil radius of the proposed discretization, for a unit grid scale, depending on the condition number of $D$ defining the second order term. In other words $\max _{1 \leq i \leq I}\left\|e_{i}\right\|$ where $D=\sum_{1 \leq i \leq I} \sigma_{i} e_{i} e_{i}^{T}$ is Selling's decomposition, see Lemma 2.3. First column : $D \in S_{2}^{++}$, second column : $D \in S_{3}^{++}$, third column : $D \in S_{3}^{++}$with the two smallest eigenvalues equal, as in (4.3). Values obtained experimentally using a fine sampling of the corresponding sets of matrices, see Theorem 1.9 for a (non-sharp) proved upper bound of the form $C_{d} \operatorname{Cond}(D)$.

Selling's algorithm [BCM16, BOZ04, BBM21, FM14] and in particular the approximation of sub-riemannian and non-holonomic eikonal equations [Mir17, Mir19]. However, this approach looses relevance for even stronger anisotropies $\operatorname{Cond}(D(x)) \gg 10$, because the numerical scheme stencils become excessively wide and accuracy therefore degrades. In this case one may limit the size of the stencils at the price of a consistency error [BOZ04, section 6], or alternatively switch to completely different techniques such as asymptotic preserving schemes [DLNN12].

For any discretization step $h>0$, we let $\Omega_{h}:=\Omega \cap h \mathbb{Z}^{d}$ and consider the finite differences scheme

$$
L_{h} u(x)=f(x) \quad \text { in } \Omega_{h},
$$

where one has, denoting $g(x, p):=\langle\omega(x), p\rangle$ in the linear case (resp. $g(x, p):=\frac{1}{2}\langle\omega(x), p\rangle^{2}$ in the quasi-linear case)

$$
-L_{h} u(x):=g\left(x, D(x)^{-1} \nabla_{h}^{D(x)} u(x)\right)+\frac{1}{2} \Delta_{h}^{D(x)} u(x) .
$$

The Dirichlet boundary condition from (4.1) does not appear in (4.4) because it is implicitly implemented via the finite differences operators, defined as (A.3) and (A.4) when the point $x$ is near $\partial \Omega$. See Appendix A for more discussion on the extension of the scheme of Definition 1.5 to non-constant coefficients, Dirichlet boundary conditions, and non-linear operators.

As announced, we present synthetic tests of our numerical scheme. For that purpose, a function $u: \bar{\Omega} \rightarrow \mathbb{R}$ is chosen with a closed form expression, and the right hand side $f: \Omega \rightarrow \mathbb{R}$ is generated by symbolic differentiation and evaluation of $\mathcal{L} u$, so that $u$ obeys (4.1) with boundary condition $g:=u_{\mid \partial \Omega}$. The discretized PDE (4.4) is then solved for a range of grid scales $h>0$, and the resulting $l^{1}\left(\Omega_{h}\right)$ and $l^{\infty}\left(\Omega_{h}\right)$ reconstruction errors are reported in Figures 3 to 6 .

The chosen exact solutions are a smooth function $\mathbf{u}_{1}$, a $C^{2,0.5}$ function $\mathbf{u}_{2}$, and a singular function $\mathbf{u}_{3}$, inspired by [FJ17] for $\mathbf{u}_{1}$ and by [FO13] for $\mathbf{u}_{2}$ and $\mathbf{u}_{3}$, and defined in $\bar{\Omega}$ by

$$
\mathbf{u}_{1}(x):=\frac{1}{4}\|x\|^{4}, \quad \mathbf{u}_{2}(x):=\max (0,\|x\|-0.4)^{2.5}, \quad \mathbf{u}_{3}(x):=\sqrt{d-\|x\|^{2}} .
$$

The multiplicative coefficient $1 / 4$ in the definition of $\mathbf{u}_{1}$ is choosen so that the range of values taken by $\left\|\nabla \mathbf{u}_{1}\right\|$ in $\Omega$ remains close to the one of values taken by $\left\|\nabla \mathbf{u}_{2}\right\|$, since the gradient magnitude influences the DDE property of the scheme (4.4) in the quasi-linear case, see $§ 4.1$. In numerical experiments, we also adjust the parameter $\mu$ in the definition of the tensor field $D: \bar{\Omega} \rightarrow S_{d}^{++}$so that DDE holds at reasonable grid scales.

Empirically we observe second order convergence $\left\|\mathbf{u}-u_{h}\right\|_{1}=\mathcal{O}\left(h^{2}\right)$ and $\left\|\mathbf{u}-u_{h}\right\|_{\infty}=\mathcal{O}\left(h^{2}\right)$, where $\mathbf{u}$ is among the two test functions $\mathbf{u}_{1}$ and $\mathbf{u}_{2}$ defined in (4.5) and $u_{h}$ is the numerical solution of (4.4) with the corresponding r.h.s. for both the linear and quasi-linear operators 
(4.2), in both dimension two and three, see Figures 3 to 6 . For the test function $\mathbf{u}_{3}$, first order convergence $\left\|\mathbf{u}_{3}-u_{h}\right\|_{1}=\mathcal{O}(h)$ and $\left\|\mathbf{u}_{3}-u_{h}\right\|_{\infty}=\mathcal{O}(h)$ is observed instead. From a theoretical standpoint, convergence was not expected for $\mathbf{u}_{3}$ and the quasi-linear scheme, since the DDE property is not guaranteed in this case, even for small $h$.

For the quasi-linear equations, a Newton method is used, converging in at most 12 iterations in our experiments with tolerance $10^{-8}$ on the max-norm of residual of the discretized PDE.

Remark 4.1 (Dominant source of numerical error). The curves of convergence associated to the linear and quasi-linear equations are conspicuously similar in several cases: for the function $\mathbf{u}_{2}$ in dimension two, see Figures 3 and 4 , and for $\mathbf{u}_{1}, \mathbf{u}_{2}$, and $\mathbf{u}_{3}$ in dimension three, see Figures 5 and 6. This suggests that the discretization of the first order term in (4.2) is not the dominant source of error in these cases.

For $\mathbf{u}_{1}$ and $\mathbf{u}_{3}$ in dimension three, we obtained a different convergence curve when changing the tensor field $D: \bar{\Omega} \rightarrow S_{d}^{++}$, suggesting that the discretization of $\operatorname{Tr}\left(D \nabla^{2} u\right)$ is the dominant source of numerical error. For the $C^{2,0.5}$ function $\mathbf{u}_{2}$, we did not observe a significative difference in the curves of convergence when changing the tensor field $D$, but we did observe one when replacing the radius $r=0.4$ with 0.5 in its definition, suggesting that the dominant source of error is related to the configuration of the grid points $\Omega_{h}$ in the vicinity of the sphere of radius $r$ across which $\mathbf{u}_{2}$ is non-smooth.

\subsection{Theoretical guarantees of Discrete Degenerate Ellipticity}

An a-priori analysis allows to guarantee the DDE property of the numerical schemes used in our numerical experiments (except in one case where it fails), thanks to the explicit and reasonably simple expression of the PDE coefficients (4.2) (and, in the quasi-linear case, of the PDE solution (4.5)). In practical applications, such an analysis may not be possible, but alternatively the DDE property can be checked numerically by looking at the sign of the coefficients of the Jacobian matrix of the discretized operator $L_{h}$.

Letting $M(x):=D(x)^{-1}$, one easily obtains

$$
\|M(x)\|=\mu^{-1}\left(3 /\left(2+\cos \left(\pi x_{1}\right)\right)\right) \nu^{-1} \leq 3 \mu^{-1} \nu^{-1},
$$

and therefore

$$
\|M(x)\|^{1 / 2}\|\omega(x)\|_{M(x)} \leq\|M(x)\|\|\omega(x)\| \leq 3 \mu^{-1} \nu^{-1} .
$$

It follows that the pair $(h \omega(x), D(x))$ is canonically feasible as soon as $h \leq c_{d} \mu \nu / 3$, where the absolute constant $c_{d}$ is specified in Theorem 1.7. The discretization of the linear operator (4.2, left) is thus DDE under these conditions.

We now check whether the discretization of the quasi-linear operator $(4.2$, right) is DDE in a neighborhood of the solutions (4.5), by linearizing the operator. For any $x \in \bar{\Omega}$ and $p \in \mathbb{R}^{d}$ one has $\left\|\nabla_{p} g(x, \nabla u(x))\right\|=\|\langle\omega(x), \nabla u(x)\rangle \omega(x)\| \leq\|\omega(x)\|^{2}\|\nabla u(x)\| \leq\|\nabla u(x)\|$. By the same resoning as above, if $\mathbf{u}$ denotes either one of the functions $\mathbf{u}_{1}$ and $\mathbf{u}_{2}$ in (4.5), then the pair $\left(h \nabla_{p} g(x, \nabla \mathbf{u}(x)), D(x)\right)$ is canonically feasible for all $x \in \Omega$, and thus the scheme (4.4) is DDE in the neighborhood of $\mathbf{u}$, as soon as

$$
h\|\nabla \mathbf{u}\|_{L^{\infty}(\Omega)}<c_{d} \mu \nu / 3 .
$$

where we used that $\nabla \mathbf{u}_{1}$ and $\nabla \mathbf{u}_{2}$ are bounded on $\Omega$. In contrast $\left\|\nabla \mathbf{u}_{3}(x)\right\|$ is unbounded when $x \rightarrow(1, \cdots, 1) \in \partial \Omega$. Thus DDE fails in the neighborhood of $\mathbf{u}_{3}$, but as noted above we do still observe convergence empirically in this particular case. 

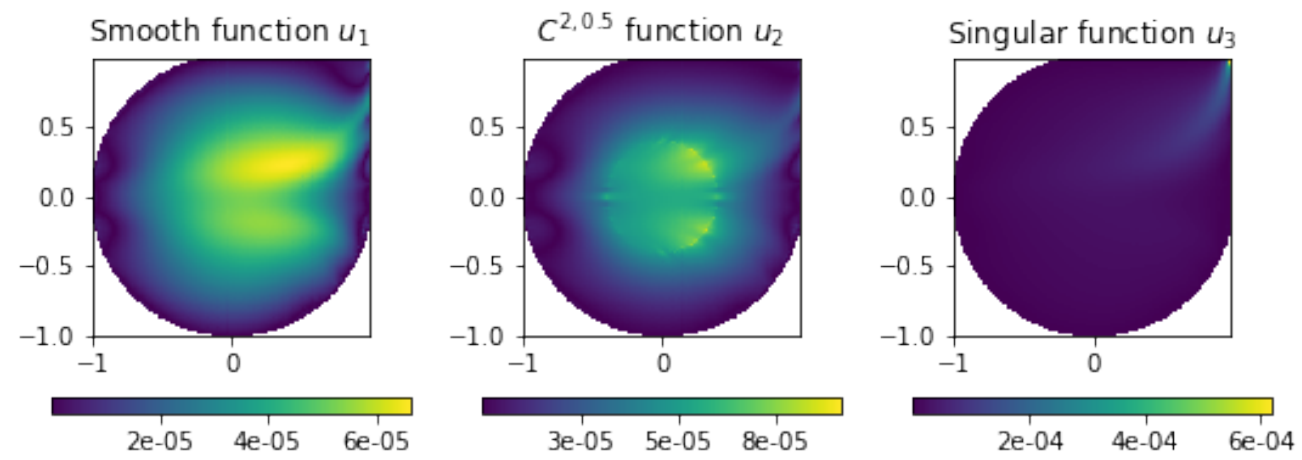

Figure 2: Errors in numerical solutions to the linear equation in dimension $d=2$, with parameters $\mu=2, \nu=1 / 10, h=1 / 100$, and with exact solutions $\mathbf{u}_{1}, \mathbf{u}_{2}$, and $\mathbf{u}_{3}$.
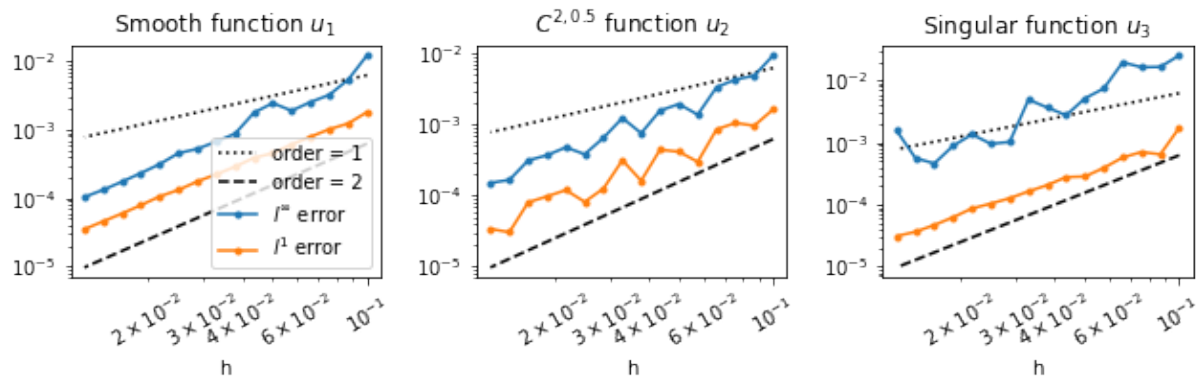

Figure 3: Convergence of the numerical scheme for the linear equation in dimension $d=2$, with parameters $\mu=2$ and $\nu=1 / 10$, and with exact solutions $\mathbf{u}_{1}, \mathbf{u}_{2}$, and $\mathbf{u}_{3}$. Degenerate ellipticity is guaranteed by $\S 4.1$ for $h \leq 1 / 30 \approx 0.0333$ and empirically observed up to $h \approx 0.0660$.
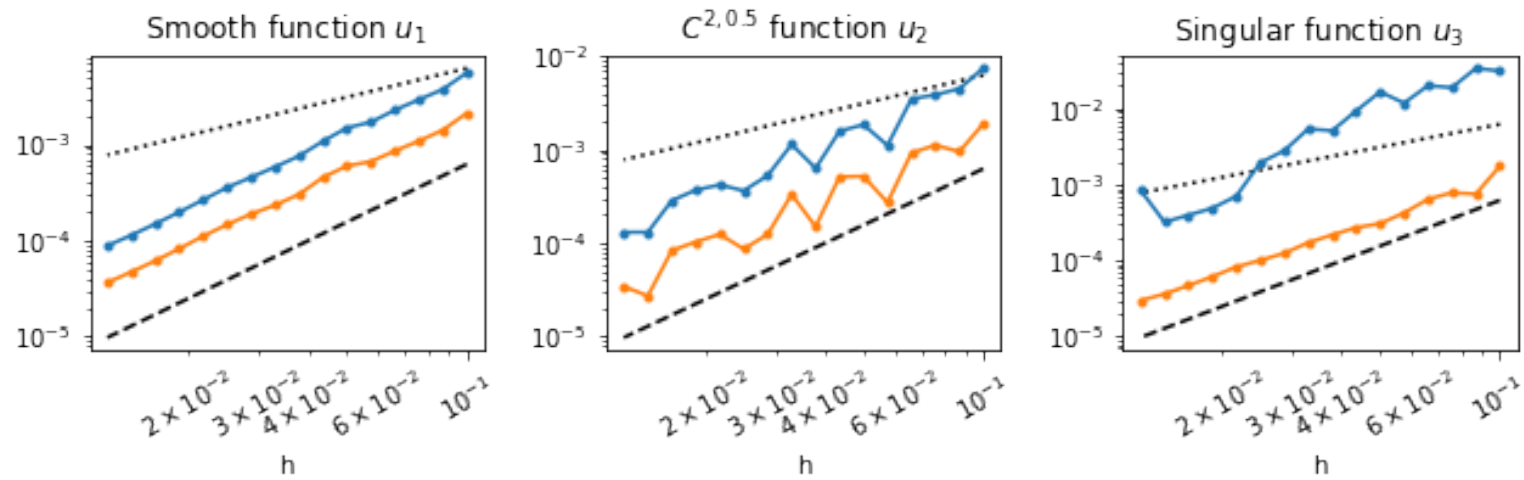

Figure 4: Convergence of the numerical scheme for the quasi-linear equation in dimension $d=2$, with parameters $\mu=4$ and $\nu=1 / 10$, and with exact solutions $\mathbf{u}_{1}, \mathbf{u}_{2}$, and $\mathbf{u}_{3}$. The legend is as in Figure 3. In the neighborhood of functions $\mathbf{u}_{1}$ and $\mathbf{u}_{2}$, degenerate ellipticity is guaranteed by $\S 4.1$ respectively for $h<1 /(30 \sqrt{2}) \approx 0.0236$ and for $h<1 /\left(75(\sqrt{2}-0.4)^{1.5}\right) \approx 0.0131$. It is observed empirically in the last iteration of the Newton method respectively up to $h \approx 0.0379$ and up to $h \approx 0.0435$. In the case of the singular function $\mathbf{u}_{3}$, degenerate ellipticity is not theoretically guaranteed, but it is nevertheless observed empirically in the last iteration of the Newton method up to $h \approx 0.0574$. 

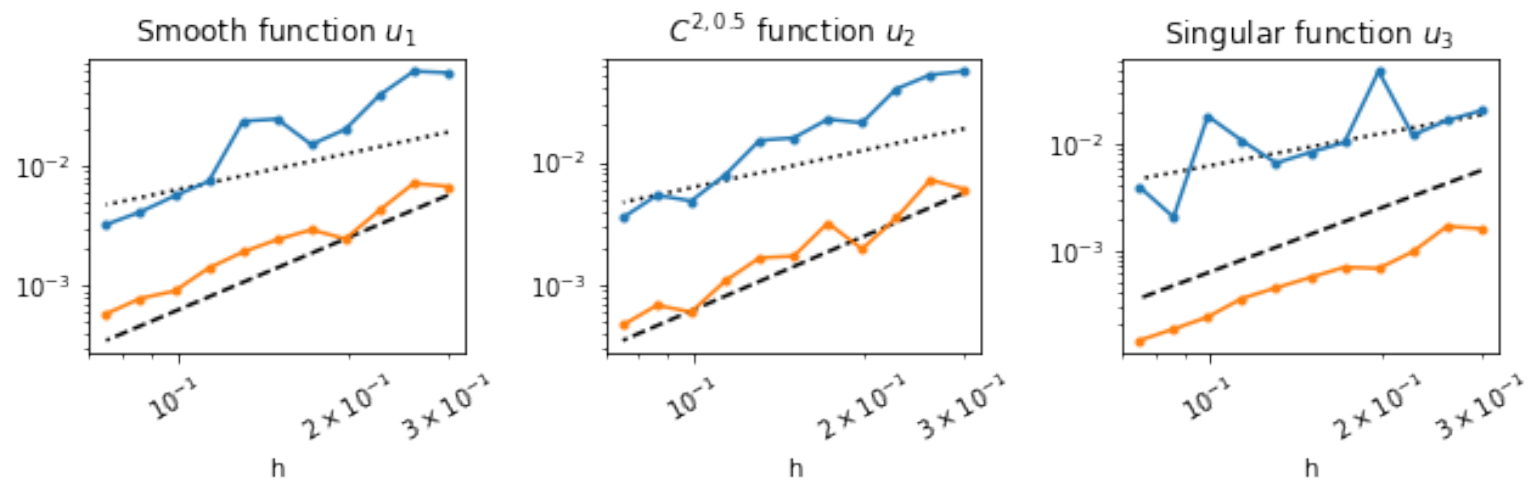

Figure 5: Convergence of the numerical scheme for the linear equation in dimension $d=3$, with parameters $\mu=8$ and $\nu=1 / 10$, and with exact solutions $\mathbf{u}_{1}, \mathbf{u}_{2}$, and $\mathbf{u}_{3}$. The legend is as in Figure 3. Degenerate ellipticity is guaranteed by $\S 4.1$ for $h \leq 1 /(5 \sqrt{3}) \approx 0.115$ and empirically observed up to $h \approx 0.198$.
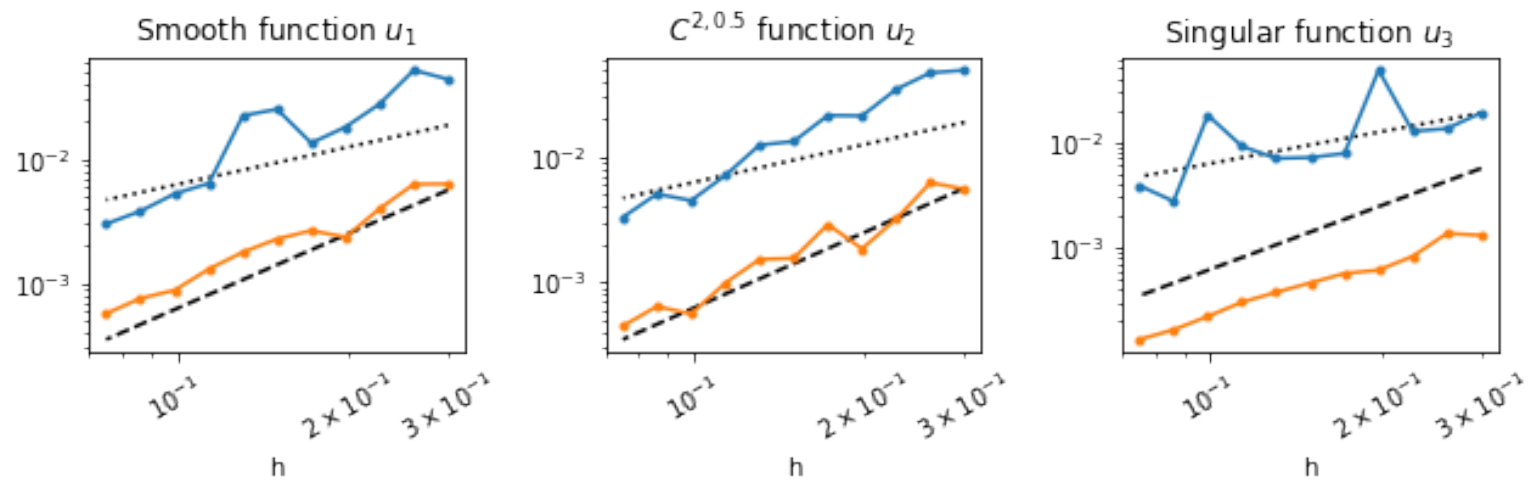

Figure 6: Convergence of the numerical scheme for the quasi-linear equation in dimension $d=3$, with parameters $\mu=16$ and $\nu=1 / 10$, and with exact solutions $\mathbf{u}_{1}, \mathbf{u}_{2}$, and $\mathbf{u}_{3}$. The legend is as in Figure 3. In the neighborhood of functions $\mathbf{u}_{1}$ and $\mathbf{u}_{2}$, degenerate ellipticity is guaranteed by $\S 4.1$ respectively for $h<2 / 135 \approx 0.0148$ and for $h<1 /\left(75 \sqrt{3}(\sqrt{3}-0.4)^{1.5}\right) \approx 0.00501$. It is observed empirically in the last iteration of the Newton method respectively up to $h \approx 0.131$ and up to $h \approx 0.261$. In the case of the singular function $\mathbf{u}_{3}$, degenerate ellipticity is not theoretically guaranteed, but it is nevertheless observed empirically in the last iteration of the Newton method up to $h=0.3$, that is, for all values of $h$ we tested in the graphs above. 


\section{Conclusion and perspectives}

In this paper, we answer whether one can discretize linear PDE operator, of order at most two and in dimension $d \leq 3$, using a second order consistent finite difference scheme obeying the discrete degenerate ellipticity property. The question is basic and of broad interest, and in dimension $d=1$ the answer is indeed simple, well known, and taught at a basic level. In dimension $d \in\{2,3\}$ however the anisotropy of the second order part of the operator comes into play, and a subtler analysis is required. Leveraging tools from the field of Euclidean lattice geometry, we could characterize whether a discretization exists, and provide an explicit (quasi-)optimal construction. Numerical experiments illustrate the efficiency of the method in dimension $d \in\{2,3\}$, on linear and quasi-linear problems.

Several research directions are open, both practical and theoretical, including (i) applications to PDEs arising from concrete problems, especially those whose first order term is large, e.g. depending on a relaxation parameter, (ii) extensions to fully non-linear HJB PDEs, and (iii) a theoretical analysis of the convergence rates. Another interesting open problem is the extension of our results for a dimension $d>3$, which is not obvious, since a key ingredient of our analysis known as $D$-obtuse superbases does not necessarily exist in that case, see Definition 2.1 and the discussion below.

\section{A Adaptation to quasi-linear and fully non-linear PDEs}

The numerical scheme presented in the introduction of this paper applies to linear schemes with constant coefficients, defined over the full space $\mathbb{R}^{d}, d \in\{2,3\}$. We illustrate in this appendix how the three restrictions in emphasis can be relaxed. For that purpose let us recall the definition of a discrete degenerate elliptic scheme, in a general setting.

Definition A.1. Let $X$ be a discrete set, and for each $x \in X$ let $V(x) \subseteq X \backslash\{x\}$ be a finite set (the neighbors, or stencil of $x$ ). Let also $\mathbb{U}:=\mathbb{R}^{X}$. A numerical scheme on $X$, with stencil $V$, is a mapping $F: \mathbb{U} \rightarrow \mathbb{U}$ of the form

$$
F u(x):=\mathcal{F}\left(x, u(x),[u(x)-u(y)]_{y \in V(x)}\right) .
$$

It is said discrete degenerate elliptic (DDE) if $\mathcal{F}$ is non-decreasing w.r.t. the second and third arguments (coordinate wise).

Definition 1.1, from the introduction, is a special case of Definition A.1, adapted to linear schemes with constant coefficients, and choosing $X=h \mathbb{Z}^{d}$ and $V(x):=\left\{x+h e_{i} ; 1 \leq|i| \leq I\right\}$. In the rest of this appendix, we show how various natural extensions of our numerical scheme fit into the general framework of Definition A.1.

\section{Non constant coefficients}

Discrete Degenerate Ellipticity is a local property, which only needs to be verified pointwise, independently at each point $x \in X$ of the discretization domain, see Definition A.1. As a result, the numerical scheme presented in this paper trivially extends to non-constant coefficients. More precisely, let $\omega$ and $D$ be a field of vectors and of symmetric positive definite matrices, and let $h>0$ be a grid scale. Then we can define the counterparts with variable coefficients of the linear PDE operator (1.1) and of its canonical discretization (1.7)

$$
\begin{aligned}
-\mathcal{L} u(x) & :=\langle\omega(x), \nabla u(x)\rangle+\frac{1}{2} \operatorname{Tr}\left(D(x) \nabla^{2} u(x)\right), \\
-L_{h} u(x) & :=\left\langle D(x)^{-1} \omega(x), \nabla_{h}^{D(x)} u(x)\right\rangle+\frac{1}{2} \Delta_{h}^{D(x)} u(x) .
\end{aligned}
$$


The scheme $L_{h}$ is DDE under the same conditions, pointwise, as in the constant coefficient case. It is not hard to show that the coefficients $x \mapsto \rho_{i}(x) \geq 0$ of $L_{h}$ expressed as in (1.1) are Lipschitz, provided $\omega$ and $D$ are Lipschitz. Interestingly, convergence rates have been established in a similar setting [Kry05] but under the slightly stronger assumption that $x \mapsto \sqrt{\rho_{i}(x)}$ is Lipchitz.

\section{Dirichlet boundary conditions}

Consider a bounded open domain $\Omega \subseteq \mathbb{R}^{d}, d \in\{2,3\}$, equipped with Dirichlet boundary conditions $f: \partial \Omega \rightarrow \mathbb{R}$, and let $\Omega_{h}:=\Omega \cap h \mathbb{Z}^{d}$, where the grid scale $h>0$ is fixed in the following. Forall $x \in \Omega_{h}, e \in \mathbb{Z}^{d} \backslash\{0\}$, define $h_{x}^{e}:=\min \left\{k>0 ; x+k e \in \Omega_{h} \cup \partial \Omega\right\}$, and note that $0<h_{x}^{e} \leq h$. Introduce the first and second finite difference operators, where for convenience we denote $h^{ \pm}:=h_{x}^{ \pm e}$, and where $u: \Omega_{h} \rightarrow \mathbb{R}$ is extended to $\partial \Omega$ using the provided Dirichlet boundary condition

$$
\begin{aligned}
\delta_{h}^{e} u(x) & :=\frac{1}{2}\left(\frac{u\left(x+h^{+} e\right)-u(x)}{h^{+}}-\frac{u\left(x-h^{-} e\right)-u(x)}{h^{-}}\right), \\
\Delta_{h}^{e} u(x) & :=\frac{2}{h^{+}+h^{-}}\left(\frac{u\left(x+h^{+} e\right)-u(x)}{h^{+}}+\frac{u\left(x-h^{-} e\right)-u(x)}{h^{-}}\right) .
\end{aligned}
$$

Note that this construction coincides with Definition 1.4 when $x$ is sufficiently far from $\partial \Omega$. For smooth $u$, one has $\delta_{h}^{e} u(x)=\langle\nabla u(x), e\rangle+\mathcal{O}\left(h^{r}\right)$ and $\Delta_{h}^{e} u(x)=\mathcal{O}\left(h^{r}\right)$ where $r=1$ if $x$ is close to $\partial \Omega_{h}$, and $r=2$ otherwise. In addition the discrete operator defined by

$$
-L_{h} u(x):=\lambda \delta_{h}^{e} u(x)+\Delta_{h}^{e} u(x)
$$

is DDE provided $h \lambda \leq 2$, similarly to the constant coefficient case, since $0<h_{x}^{ \pm e} \leq h$. Therefore (A.3) and (A.4) can be used as a drop in replacement for the finite difference operators of Definition 1.4 when Dirichlet boundary conditions are used, the resulting scheme is DDE under the same conditions. More complex boundary conditions may require ad-hoc treatment.

\section{Quasi-linear operators}

Let $D \in S_{d}^{++}, d \in\{2,3\}$, and let $g: \mathbb{R}^{d} \rightarrow \mathbb{R}$ be a smooth function. Consider the quasi-linear operator $\mathcal{L}$ and its discretization $L_{h}$ defined by

$$
-\mathcal{L} u(x):=g(\nabla u(x))+\frac{1}{2} \operatorname{Tr}\left(D \nabla^{2} u(x)\right), \quad-L_{h} u(x):=g\left(D^{-1} \nabla_{h}^{D} u(x)\right)+\frac{1}{2} \Delta_{h}^{D} u(x) .
$$

The operator $\mathcal{L}$ is degenerate elliptic, since in the continuous setting this property is independent of the first order term of the PDE. On the other hand, the scheme $L_{h}$ is DDE provided the linear scheme $\tilde{L}_{h}$ defined by $-\tilde{L}_{h} u(x):=\left\langle D^{-1} \omega, \nabla_{h}^{D} u(x)\right\rangle+\frac{1}{2} \Delta_{h}^{D} u(x)$ is DDE for all $\omega \in \nabla g\left(\mathbb{R}^{d}\right)=$ $\left\{\nabla g(x) ; x \in \mathbb{R}^{d}\right\}$. (This is a severe restriction if $g$ is e.g. a quadratic function, but for such applications it can be enough to check that the scheme is DDE in a neighborhood of the solution.)

\section{Fully non-linear operators}

Fully non-linear Hamilton-Jacobi-Bellman (HJB) operators can be expressed, under mild regularity assumptions, in Isaacs form

$$
\mathcal{L} u(x):=\sup _{\alpha \in A} \inf _{\beta \in B} \mathcal{L}_{\alpha \beta} u(x)
$$


where $A, B$ are known as the control sets. In addition $\mathcal{L}_{\alpha \beta}$ is a linear degenerate elliptic operator, for all $\alpha \in A, \beta \in B$,

$$
\mathcal{L}_{\alpha \beta} u(x):=\mu_{\alpha \beta}(x)+\lambda_{\alpha \beta}(x) u(x)+\left\langle\omega_{\alpha \beta}(x), \nabla u(x)\right\rangle-\frac{1}{2} \operatorname{Tr}\left(D_{\alpha \beta}(x) \nabla^{2} u(x)\right),
$$

where $\mu_{\alpha \beta}(x) \in \mathbb{R}, \lambda_{\alpha \beta}(x) \geq 0, \omega_{\alpha \beta}(x) \in \mathbb{R}^{d}$, and $D_{\alpha \beta}(x) \in S_{d}^{+}$. In the special case where one of the sets $A$ or $B$ is a singleton, which is common (consider the Monge-Ampere [BCM16] or Pucci [BBM21] equations), then (A.5) is known as the Bellman form of the operator.

It is in principle possible to introduce samples $A_{h} \subseteq A$ and $B_{h} \subseteq B$ of the control sets, and to construct a discretization $L_{\alpha \beta}^{h}$ of each linear operator $\mathcal{L}_{\alpha \beta}$ following the approach presented in this paper. This produces a DDE approximation of the operator $\mathcal{L}$

$$
L_{h} u(x):=\sup _{\alpha \in A_{h}} \inf _{\beta \in B_{h}} L_{\alpha \beta}^{h} u(x) .
$$

Let us acknowledge, however, that this construction is far from straightforward to put in practice, especially if the sets $A$ and $B$ are non-compact, and if the condition number of the matrices $D_{\alpha \beta}(x)$ is not uniformly bounded.

\section{B Terminology and elementary properties of polyhedra}

In this section, we recall some of the terminology and elementary properties related with polyhedra, limiting our attention to those which are immediately useful in the study of Ryskov's polyhedron and its variant $\S 2.2$ and $\S 3.1$. See [BG15] for a more complete reference.

\section{B.1 Regularity and skeleton}

Definition B.1. A polyhedron in $\mathbb{R}^{n}$ is a set of the form

$$
\mathcal{M}:=\left\{x \in \mathbb{R}^{n} ; \forall i \in I,\left\langle l_{i}, x\right\rangle \geq \alpha_{i}\right\},
$$

where $l_{i} \in \mathbb{R}^{n}, \alpha_{i} \in \mathbb{R}$, and $I$ is a finite or countable set. The polyhedron $\mathcal{M}$ is said regular iff it (i) has a non-empty interior, (ii) does not contain any affine line, and (iii) can be locally described by the constraints corresponding to a finite subset of $I$.

By definition, a polyhedron is thus a convex set. Condition (ii) can be reformulated as $\operatorname{Span}\left\{l_{i} ; i \in I\right\}=\mathbb{R}^{n}$. Condition (iii) can be reformulated as follows: for all $x \in \mathcal{M}$ there exists a positive radius $r>0$ and a finite subset $I_{0} \subseteq I$ such that

$$
\left\langle l_{i}, y\right\rangle>\alpha_{i}, \forall i \in I \backslash I_{0}, \forall y \in B(x, r) .
$$

Definition B.2. Let $\mathcal{M}$ be a regular polyhedron, defined as in (B.1). A $k$-facet of $\mathcal{M}$, where $1 \leq k \leq n$, is a non-empty subset of $\mathcal{M}$ of the form

$$
\left\{x \in \mathcal{M} ; \forall i \in J,\left\langle l_{i}, x\right\rangle=\alpha_{i}\right\}, \quad \text { where } \quad \operatorname{dim} \operatorname{Span}\left\{l_{i} ; i \in J\right\}=n-k,
$$

and where $J \subseteq I$ denotes a subset of the constraint indices.

By construction, a $k$-facet is a convex subset of $\partial \mathcal{M}$ of affine dimension $k$. If a $k$-facet satisfies $\#(J)>n-k$, where $J \subseteq I$ is chosen maximal for inclusion, then it is said degenerate. By construction 0-facets are singletons, and their single point is called a vertex. On the other hand 1-facets are known as edges and come in two flavors 
- Bounded edges, of the form $\left[x_{1}, x_{2}\right]:=\left\{(1-t) x_{1}+t x_{2} ; 0 \leq t \leq 1\right\}$, where $x_{1}$ and $x_{2}$ are vertices.

- Unbounded edges, of the form $\{x+\lambda v ; \lambda \geq 0\}$, where $x$ is a vertex, and $v \in \mathbb{R}^{n} \backslash\{0\}$ is called the unbounded edge direction (unique up to multiplication by a positive constant).

Note that doubly unbounded edges, of the form $\{x+\lambda v ; \lambda \in \mathbb{R}\}$, are affine lines and are thus excluded by Definition B.1.

Remark B.3. Let $\mathcal{M}$ be a regular polyhedron, in the sense of Definition B.1. An element $x \in \mathcal{M}$ is a vertex iff $\mathbb{R}^{n}=\operatorname{Span}\left\{l_{i} ; i \in I,\left\langle l_{i}, x\right\rangle=\alpha_{i}\right\}$.

\section{B.2 Linear programs}

Linear programs are defined as the optimization of a linear functional over a polytope. A fundamental result of operational research, is that such problems can under suitable assumptions be solved by a greedy search over the graph defined by the edges of the polytope, such as the simplex algorithm [BG15]. Since Definition B.1 allows for infinitely many constraints, which is slightly more general than the common setting, we establish in Proposition B.4 a basic result on such programs, used in $§ 3.2$. Note that the infima in (B.2) may not be attained.

Proposition B.4. Let $\mathcal{M}$ be a regular polyhedron. Then for any $l \in \mathbb{R}^{n}$

$$
\begin{aligned}
& \inf \{\langle l, x\rangle ; x \in \mathcal{M}\} \\
& =\left\{\begin{array}{l}
-\infty \text { if }\langle l, v\rangle<0 \text { for some unbounded edge direction } v, \\
\inf \{\langle l, x\rangle ; x \text { vertex of } \mathcal{M}\} \text { otherwise. }
\end{array}\right.
\end{aligned}
$$

Proof. By point (i) of Definition B.1, there exists $x_{*} \in \operatorname{int}(\mathcal{M})$. By point (ii) of Definition B.1, one has $\operatorname{Span}\left\{l_{i} ; i \in I\right\}=\mathbb{R}^{n}$, otherwise $x_{*}+\mathbb{R} v$ is an affine line contained in $\mathcal{M}$ for any non-zero $v \in \operatorname{Span}\left\{l_{i} ; i \in I\right\}^{\perp}$, hence there exists $I_{*} \subseteq I$ with $\#\left(I_{*}\right)=n$ and such that $\left(l_{i}\right)_{i \in I_{*}}$ is a basis of $\mathbb{R}^{n}$.

Define $l_{*}:=\sum_{i \in I_{*}} l_{i}$, and consider for each $\alpha>l_{*}\left(x_{*}\right)$ the set $\mathcal{M}_{\alpha}:=\left\{x \in \mathcal{M} ;\left\langle l_{*}, x\right\rangle \leq \alpha\right\}$. Note that for each $x \in \mathcal{M}_{\alpha}$ and $i \in I_{*}$ one has $0 \leq l_{i}(x)-\alpha_{i} \leq \alpha-\sum_{i \in I_{*}} \alpha_{i}$, hence $\mathcal{M}_{\alpha}$ is bounded. Thus $\mathcal{M}_{\alpha}$ is a compact polyhedron with non-empty interior, which by Definition B.1 (iii) is characterized by finitely many linear constraints. By Carathéodory's theorem, $\min \left\{\langle l, x\rangle ; x \in \mathcal{M}_{\alpha}\right\}$ is attained at a vertex of $\mathcal{M}_{\alpha}$, which by construction is either a vertex of $\mathcal{M}$ or the intersection of an edge of $\mathcal{M}$ (bounded or not) with the hyperplane $\left\{x \in \mathbb{R}^{n} ;\left\langle l_{*}, x\right\rangle=\alpha\right\}$. From this point, and noting that $\mathcal{M}=\cup_{\alpha \in \mathbb{R}} \mathcal{M}_{\alpha}$, the announced result easily follows.

Definition B.5 (Karush-Kuhn-Tucker relations). A set of KKT relations for $l$ in $\mathbb{R}^{n}$ and $x$ in $\mathcal{M}$ is a finitely supported family of non-negative coefficients $\left(\lambda_{i}\right)_{i \in I}$ such that

$$
l=\sum_{i \in I} \lambda_{i} l_{i}, \quad \text { and } \forall i \in I, \lambda_{i}=0 \text { or }\left\langle l_{i}, x\right\rangle=\alpha_{i} .
$$

It is known [BG15] that a linear form $l \in \mathbb{R}^{n}$ attains its minimum at a given point $x$ of a regular polyhedron $\mathcal{M}$, if and only if there exists KKT relations for $l$ and $x$. The next result establishes a uniqueness property of the KKT relations.

Proposition B.6. Let $\mathcal{M} \subseteq \mathbb{R}^{n}$ be a regular polyhedron, in the sense of Definition B.1. Assume that one has a set of $K K T$ relations $\left(\lambda_{i}\right)_{i \in I}$ for some $l \in \mathbb{R}^{d}$ at a non-degenerate vertex $x \in \mathcal{M}$. Then any other KKT relations $\left(\lambda_{i}^{\prime}\right)_{i \in I}$ at some $x^{\prime} \in \mathcal{M}$ (possibly distinct from $x$ ), for the same $l$, obey $\lambda_{i}=\lambda_{i}^{\prime}$ for all $i \in I$. 
Proof. For all $i \in I$ such that $\lambda_{i}^{\prime}>0$ one has $\left\langle l_{i}, x^{\prime}\right\rangle=\alpha_{i}$, thus $\left\langle l_{i}, x-x^{\prime}\right\rangle \geq 0$. On the other hand one has $\langle l, x\rangle=\left\langle l, x^{\prime}\right\rangle=\inf \{\langle l, z\rangle ; z \in \mathcal{M}\}$, and therefore $0=\left\langle l, x-x^{\prime}\right\rangle=\sum_{i \in I} \lambda_{i}^{\prime}\left\langle l_{i}, x-x^{\prime}\right\rangle$. Combining these two arguments we obtain that for all $i \in I$ such that $\lambda_{i}^{\prime}>0$ one has $\left\langle l_{i}, x-x^{\prime}\right\rangle=$ 0 , and therefore $\left\langle l_{i}, x\right\rangle=\alpha_{i}$. Since $x$ is a non-degenerate vertex, the family $\left\{l_{i} ; i \in I,\left\langle l_{i}, x\right\rangle=\alpha_{i}\right\}$ is a basis of $\mathbb{R}^{n}$, which implies the announced uniqueness result.

\section{B.3 Edges originating from a vertex}

In this section, we present a constructive enumeration of all the edges of a regular polyhedron $\mathcal{M}$ containing a given vertex $x$. This description follows from Definition B.2 of $k$-facets, here with $k=1$. We use the notations of Definition B.1.

Let $J:=\left\{i \in I ;\left\langle l_{i}, x\right\rangle=\alpha_{i}\right\}$ denote the indices of all the active constraints at the vertex $x$ of $\mathcal{M}$. In order to enumerate all the edges of $\mathcal{M}$ containing $x$, bounded or unbounded, the steps are the following:

(A) Consider successively all subsets $S$ of $J$ with cardinality $n-1$.

(B) If $\operatorname{dim} \operatorname{Span}\left\{l_{i} ; i \in S\right\}<n-1$, then skip this subset. Otherwise denote by $\nu \in \mathbb{R}^{n} \backslash\{0\}$ the vector, which is unique up to a scalar multiplication, such that $\left\langle l_{i}, \nu\right\rangle=0$ for all $i \in S$.

(C) Replace $\nu$ with its opposite $-\nu$, if necessary, in such way that $\left\langle l_{i}, \nu\right\rangle \geq 0$ for all $i \in J \backslash S$. If that is not possible, then skip this subset.

(D) Compute $\Lambda:=\sup \{\lambda \in \mathbb{R} ; x+\lambda \nu \in \mathcal{M}\}$. If $\Lambda=+\infty$, then there is an unbounded edge at $x$ in the direction of $\nu$. Otherwise, $x$ and $x+\Lambda \nu$ are the vertices of a bounded edge of $\mathcal{M}$.

\section{References}

[BBM21] J. F. Bonnans, G. Bonnet, and J.-M. Mirebeau, Monotone and second order consistent scheme for the two dimensional Pucci equation, Numerical Mathematics and Advanced Applications ENUMATH 2019 (F. J. Vermolen and C. Vuik, eds.), Springer International Publishing, to appear, 2021.

[BCM16] J.-D. Benamou, F. Collino, and J.-M. Mirebeau, Monotone and consistent discretization of the Monge-Ampère operator, Mathematics of Computation 85 (2016), no. 302, $2743-2775$.

[BG15] J. F. Bonnans and S. Gaubert, Recherche opérationnelle, Aspects mathématiques et applications, Les Éditions de l'École polytechnique, 2015.

[BOZ04] J. F. Bonnans, É. Ottenwaelter, and H. Zidani, A fast algorithm for the two dimensional HJB equation of stochastic control, ESAIM: Mathematical Modelling and Numerical Analysis 38 (2004), no. 4, 723-735.

[CIL92] M. G. Crandall, H. Ishii, and P.-L. Lions, User's guide to viscosity solutions of second order partial differential equations, Bulletin of the American Mathematical Society 27 (1992), no. 1, 1-67.

[CS88] J. H. Conway and N. J. A. Sloane, Low-dimensional lattices. III. Perfect forms, Proceedings of the Royal Society of London A: Mathematical, Physical and Engineering Sciences 418 (1988), no. 1854, 43-80. 
[CS92] _ Low-dimensional lattices. VI. Voronoi reduction of three-dimensional lattices, Proceedings of the Royal Society A: Mathematical, Physical and Engineering Sciences 436 (1992), no. 1896, 55-68.

[DK09] RONALD DEVORE and Angela Kunoth (eds.), Multiscale, Nonlinear and Adaptive Approximation, Springer Berlin Heidelberg, Berlin, Heidelberg, 2009.

[DLNN12] Pierre Degond, Alexei Lozinski, Jacek Narski, and Claudia Negulescu, An asymptotic-preserving method for highly anisotropic elliptic equations based on a micro-macro decomposition, Journal of Computational Physics 231 (2012), no. 7, $2724-2740$.

[DSSV12] M. Dutour Sikirić, A. Schürmann, and F. Vallentin, Inhomogeneous extreme forms, Annales de l'institut Fourier, 2012, pp. 2227-2255.

[Erd92] R. Erdahl, A cone of inhomogeneous second-order polynomials, Discrete and Computational Geometry 8 (1992), no. 4, 387-416.

[FJ17] X. Feng and M. Jensen, Convergent semi-Lagrangian methods for the Monge-Ampère equation on unstructured grids, SIAM Journal on Numerical Analysis 55 (2017), no. 2, $691-712$.

[FM14] J. Fehrenbach and J.-M. Mirebeau, Sparse non-negative stencils for anisotropic diffusion, Journal of Mathematical Imaging and Vision 49 (2014), no. 1, 123-147.

[FO11] B. D. Froese and A. M. Oberman, Convergent finite difference solvers for viscosity solutions of the elliptic Monge-Ampère equation in dimensions two and higher, SIAM Journal on Numerical Analysis 49 (2011), no. 4, 1692-1714.

[FO13] Convergent filtered schemes for the Monge-Ampère partial differential equation, SIAM Journal on Numerical Analysis 51 (2013), no. 1, 423-444.

[Kry05] N. V. Krylov, The rate of convergence of finite-difference approximations for Bellman equations with Lipschitz coefficients, Applied Mathematics and Optimization 52 (2005), no. 3, 365-399.

[LN18] W. Li and R. H. Nochetto, Optimal pointwise error estimates for two-scale methods for the Monge-Ampère equation, SIAM Journal on Numerical Analysis 56 (2018), no. $3,1915-1941$.

[Mir16] J.-M. Mirebeau, Minimal stencils for discretizations of anisotropic PDEs preserving causality or the maximum principle, SIAM Journal on Numerical Analysis 54 (2016), no. $3,1582-1611$.

[Mir17]__ Fast-marching methods for curvature penalized shortest paths, Journal of Mathematical Imaging and Vision (2017), 1-32.

[Mir19]—, Riemannian fast-marching on cartesian grids, using Voronoi's first reduction of quadratic forms, SIAM Journal on Numerical Analysis 57 (2019), no. 6, 2608-2655.

[NS04] P. Q. Nguyen and D. Stehlé, Low-dimensional lattice basis reduction revisited, ANTS (Ducan Buell, ed.), Springer, 2004, pp. 338-357. 
[Obe06] A. M. Oberman, Convergent difference schemes for degenerate elliptic and parabolic equations: Hamilton-Jacobi equations and free boundary problems, SIAM Journal on Numerical Analysis 44 (2006), no. 2, 879-895.

$\left[\mathrm{PCC}^{+} 19\right]$ S. Parisotto, L. Calatroni, M. Caliari, C.-B. Schönlieb, and J. Weickert, Anisotropic osmosis filtering for shadow removal in images, Inverse Problems 35 (2019), no. 5, 054001.

[Sch09a] A. Schürmann, Computational geometry of positive definite quadratic forms, University Lecture Series 49 (2009).

[Sch09b] _ Enumerating perfect forms, Contemporary Mathematics 493 (2009), 359.

[Sel74] E. Selling, Ueber die binären und ternären quadratischen Formen, Journal fur die Reine und Angewandte Mathematik 77 (1874), 143-229.

[SSV07] M. Sikirić, A. Schürmann, and F. Vallentin, Classification of eight-dimensional perfect forms, Electronic Research Announcements of the American Mathematical Society 13 (2007), no. 3, 21-32.

[Vor08] G. Voronoi, Sur quelques propriétés des formes quadratiques positives parfaites, J. reine angew. Math, 1908. 